\title{
Higher rank FZZ-dualities
}

\section{Thomas Creutzig and Yasuaki Hikida}

Department of Mathematical and Statistical Sciences, University of Alberta, Edmonton, AB T6G 2G1, Canada

Center for Gravitational Physics, Yukawa Institute for Theoretical Physics, Kyoto University, Kyoto 606-8502, Japan

E-mail: creutzig@ualberta.ca, yhikida@yukawa.kyoto-u.ac.jp

ABSTRACT: We examine strong/weak dualities in two dimensional conformal field theories by generalizing the Fateev-Zamolodchikov-Zamolodchikov (FZZ-)duality between Witten's cigar model described by the $\mathfrak{s l}(2) / \mathfrak{u}(1)$ coset and sine-Liouville theory. In a previous work, a proof of the FZZ-duality was provided by applying the reduction method from $\mathfrak{s l}(2)$ WessZumino-Novikov-Witten model to Liouville field theory and the self-duality of Liouville field theory. In this paper, we work with the coset model of the type $\mathfrak{s l}(N+1) /(\mathfrak{s l}(N) \times \mathfrak{u}(1))$ and investigate the equivalence to a theory with an $\mathfrak{s l}(N+1 \mid N)$ structure. We derive the duality explicitly for $N=2,3$ by applying recent works on the reduction method extended for $\mathfrak{s l}(N)$ and the self-duality of Toda field theory. Our results can be regarded as a conformal field theoretic derivation of the duality of the Gaiotto-Rapčák corner vertex operator algebras $Y_{0, N, N+1}[\psi]$ and $Y_{N, 0, N+1}\left[\psi^{-1}\right]$.

Keywords: Conformal and W Symmetry, Conformal Field Theory, String Duality

ARXIV EPRINT: 2010.14681 


\section{Contents}

1 Introduction 1

2 Symmetry algebra $\quad 4$

2.1 Truncation of $\mathrm{W}_{\infty}$-algebra 5

$\begin{array}{lll}2.2 & \text { Intersection of kernels of screening charges } & 6\end{array}$

3 Coset model $\quad 7$

3.1 First order formulation of WZNW model 8

3.2 First order formulation of coset model 9

$\begin{array}{lll}3.3 & \text { Spectral flow } & 10\end{array}$

4 A generalized FZZ-duality 11

4.1 Reduction from WZNW model 11

$\begin{array}{lll}4.2 & \text { Reduction from coset theory } & 14\end{array}$

$\begin{array}{lll}4.3 & \text { Dual theory } & 15\end{array}$

$\begin{array}{lll}5 & \text { A higher rank generalization } & 17\end{array}$

$\begin{array}{ll}\text { 5.1 First order formulation of coset model } & 17\end{array}$

$\begin{array}{ll}5.2 & \text { Reduction from coset theory } \\ 5.3 & 19\end{array}$

$\begin{array}{lll}5.3 & \text { Interpretation as extended BP-theory } & 21\end{array}$

5.4 Reduction from extended BP-theory 24

6 Conclusion and discussions $\quad 26$

$\begin{array}{lr}\text { A Supersymmetric dualities } & 28\end{array}$

$\begin{array}{ll}\text { B Free field realizations of BP-algebra } & 29\end{array}$

\section{Introduction}

In this paper, we examine strong/weak dualities in two dimensional conformal field theories (CFTs). In general, strong/weak dualities are quite useful, for instance, to examine strongly coupled physics from weakly coupled theories. A rare solvable example was proposed by Fateev-Zamolodchikov-Zamolodchikov (FZZ) [1], and their duality states an equivalence between Witten's cigar model [2] and so-called sine-Liouville theory. The cigar model can be described by the coset

$$
\frac{\mathfrak{s l}(2)_{k}}{\mathfrak{u}(1)}
$$


The level $k$ is related to the inverse of curvature and the sigma model description is suitable for large $k$. On the other hand, the sine-Liouville theory is described by a bosonic field $\phi$ with background charge and another bosonic field $X$ with $X \sim X+2 \pi \sqrt{k}$. The theory includes interaction terms

$$
V_{\mathrm{int}}=2 \pi \lambda\left(e^{\phi / b_{(2)}+i \sqrt{k} \tilde{X}}+e^{\phi / b_{(2)}-i \sqrt{k} \tilde{X}}\right),
$$

where $b_{(2)}=1 / \sqrt{k-2}$ and $\tilde{X}$ represents the dual of $X$. The interaction terms do not grow rapidly when $b_{(2)}$ is large enough. From this, we can see that the FZZ-duality is an example of strong/weak duality. See [3] for more details of the FZZ-duality and its application to holography.

In this paper, we generalize the FZZ-duality by replacing the coset model (1.1) with

$$
\frac{\mathfrak{s l}(N+1)_{k}}{\mathfrak{s l}(N)_{k} \times \mathfrak{u}(1)}
$$

The central charge is

$$
c=\frac{k\left((N+1)^{2}-1\right)}{k-N-1}-\frac{k\left(N^{2}-1\right)}{k-N}-1 .
$$

The symmetry of the coset model (1.3) is given by a one parameter quotient of the $\mathrm{W}_{\infty^{-}}$ algebra [4]. Recently, $\mathrm{W}$-algebras and especially the $\mathrm{W}_{\infty}$-algebra play important roles in theoretical physics. For instance, subsectors of four dimensional gauge theories are known to be described by Toda field theories with certain W-algebra symmetry [5, 6]. Moreover, the coset model (1.3) is essentially the one appearing in the higher spin holography [7] or its supersymmetric extension [8].

This connection to the cosets relevant to higher spin (super-)gravity is our first main motivation. The second motivation is a conformal field theory derivation of dualities and trialities of corner vertex operator algebras (VOAs). Recall that at the corner of interfaces of four dimensional supersymmetric gauge theories with gauge groups $\mathrm{U}\left(N_{1}\right), \mathrm{U}\left(N_{2}\right)$ and $\mathrm{U}\left(N_{3}\right)$ there is a VOA, called the $Y_{N_{1}, N_{2}, N_{3}}$-algebra [9]. This vertex algebra is parameterized by a parameter $\psi$ (for the associated CFT it is the inverse of the coupling constant), which is the level of the associated $\mathrm{W}$-algebra shifted by the dual Coxeter number. ${ }^{1}$

The triality conjecture of [9] asserts that $Y_{N_{1}, N_{2}, N_{3}}$-algebras are invariant under permutations of the labels where the coupling constant also changes appropriately. Especially the $Y_{N_{1}, N_{2}, N_{3}}$-algebra at coupling $\psi$ coincides conjecturally with the $Y_{N_{2}, N_{1}, N_{3}}$-algebra at coupling $\psi^{-1}$. I.e. if one of the theories is strongly coupled (small $\psi$ ) the other one is weakly coupled (large $\psi$ ). The triality conjecture is a theorem if one of the labels is zero [4]. The simplest example, the $Y_{N, 0,0}$-algebra is the $\mathrm{W}_{N}$-algebra of $\mathfrak{s l}(N)$ and the isomorphism between $Y_{N, 0,0}$-algebra and $Y_{0, N, 0}$-algebra is Feigin-Frenkel duality [11], while the isomorphism between $Y_{N, 0,0}$-algebra and $Y_{0,0, N}$-algebra is the coset realization of the

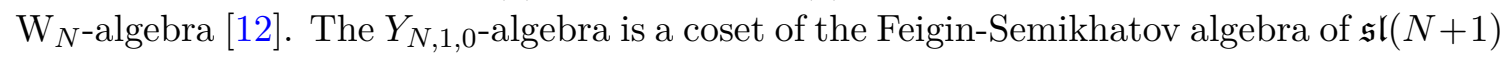
and the isomorphism between $Y_{N, 1,0}$-algebra and $Y_{1, N, 0}$-algebra is the Feigin-Semikhatov

\footnotetext{
${ }^{1} \mathrm{~W}$-algebras are usually defined as quantum Hamiltonian reductions, that is certain BRST-cohomologies, of affine vertex algebras. The level of the $\mathrm{W}$-algebra is the level of the affine vertex algebra and this was first introduced in generality in [10].
} 
duality [13], proven in [14]. It is expected that the dualities and trialities of the vertex algebras of protected operators at corners and junctions lift to dualities of spaces of conformal blocks and thus providing a picture that contains the quantum geometric Langlands correspondence [15-17].

It is thus desirable to give conformal field theory derivations of the dualities and trialities of $Y$-algebras. Especially, one would like to not only have an isomorphism of VOAs, but additional correspondences as e.g. a matching of correlation functions. Our higher rank FZZ-duality is precisely this for the case of the $Y_{0, N, N+1}$-algebras. Moreover, the $Y_{N_{1}, N_{2}, N_{3}}$-algebras are conjecturally the same as the $W_{N_{1}, N_{2}, N_{3}}$-algebras of Bershtein, Feigin and Merzon [18], see also [19, 20]. The latter act on the cohomology of moduli spaces of spiked instantons of Nekrasov [21]. It is thus also desirable to get a conformal field theory perspective on the conjectural relation between $Y_{N_{1}, N_{2}, N_{3}}$-algebras and $W_{N_{1}, N_{2}, N_{3}}$-algebras. The latter are characterized as intersections of kernels of screening charges acting on a free field theory, i.e. they have a nice description in terms of a theory with free kinetic terms and interaction terms. The dual theory that we get is a theory with free kinetic terms and interaction terms and the holomorphic part of our interaction terms are precisely the screening charges of the $W_{N, 0, N+1}$-algebra [20], i.e. our formalism naturally relates $Y_{0, N, N+1}$-algebra to $W_{N, 0, N+1}$-algebra.

The $W_{N, 0, N+1}$-algebra is called as $\mathrm{W}(\mathfrak{s l}(N+1 \mid N))$-algebra in $[18,19]$ due to its $\mathfrak{s l}(N+$ $1 \mid N)$ structure. The $\mathrm{W}(\mathfrak{s l}(N+1 \mid N))$-algebra is closely related to the $\mathcal{N}=2 \mathrm{~W}_{N+1}$-algebra, which is obtained as a Hamiltonian reduction of $\mathfrak{s l}(N+1 \mid N)$ current algebra. However, note that the two algebras are different. The $\mathcal{N}=2 \mathrm{~W}_{N+1}$-algebra is identified as the symmetry algebra of $\mathfrak{s l}(N+1 \mid N)$ Toda field theory, and it is believed to be realized by the $\mathrm{CP}_{N}$ Kazama-Suzuki coset model as well $[22,23] .{ }^{2}$ From this fact, we can also think of a supersymmetric version of our higher rank FZZ-duality, but its detailed analysis is postponed to future work.

In this paper, we explicitly derive the duality with the coset model (1.3) for $N=2,3$ by extending the proof of the original FZZ-duality in [26]. In the proof of FZZ-duality, the $\mathfrak{s l}(2)$ factor of the coset (1.1) described by $\mathfrak{s l}(2)$ Wess-Zumino-Novikov-Witten (WZNW) model is reduced to Liouville field theory by applying the analysis of [27], which is a path integral derivation of (generalized) Ribault-Teschner relation [28, 29]. We further utilize the self-duality of Liouville field theory, which is the key point for the nature of strong/weak duality. There have been several works on generalizations of the reduction method [3032]. Recently, big progress was made in [33], and the result enables us to do the current generalizations of FZZ-duality. In order to directly apply the procedure of [26], we may want to reduce the $\mathfrak{s l}(N+1)$ factor of the coset (1.3) to $\mathfrak{s l}(N+1)$ Toda field theory. If this is possible, then the self-duality of the Toda field theory can be applied. However, its difficulty has been recognized, see, e.g., [32, 34]. We can avoid this difficulty by not directly reducing the WZNW model to the Toda field theory but instead using intermediate theories with non-regular type of $\mathrm{W}$-algebra symmetry [33]. A famous example of non-regular $\mathrm{W}$-algebra

\footnotetext{
${ }^{2}$ This Conjecture is proven in the case $N=2$ [24] and by Lemma 7.12 of [25] both theories have the same type of minimal strong generators.
} 
is given by Bershadsky-Polyakov (BP-)algebra [35, 36]. We make use of the intermediate theories in the following way. The method of [27] and its generalizations heavily rely on the free field realizations of algebras. For non-regular W-algebras, it was noticed that several free field realizations are available [37, 38]. The reduction then becomes possible by replacing its free field realization with a convenient one for the intermediate theories. Throughout this paper, we assume that the theories are defined on a genus zero Riemann sphere. It is an interesting open problem to deal with theories on more generic surfaces.

The paper is organized as follows. In the next section, we start by examining the symmetry of the coset model, which is also underlying the duality. In section 3, we study the coset model (1.3) with $N=2$. We develop a free field realization of the coset algebra by following [39, 40], which is necessary to apply the reduction method of [27, 33]. In section 4, we derive a generalized FZZ-duality with the coset (1.3) for $N=2$. In this case, the annoying issue associated with higher rank algebra does not arise and the original reduction method of [27] and the self-duality of $\mathfrak{s l}(3)$ Toda field theory can be applied straightforwardly. Massaging the theory obtained in this way, we derive a duality between the coset model (1.3) with $N=2$ and a generalized sine-Liouville theory with an $\mathfrak{s l}(3 \mid 2)$ structure. In section 5 , we generalize the analysis by considering the coset model (1.3) with $N=3$. A free field realization of the coset algebra can be obtained in a way similar to the $N=2$ case. However, the original reduction method of [27] cannot be applied directly. We overcome this difficulty by utilizing the technique of [33] briefly explained above and derive the dual theory with an $\mathfrak{s l}(4 \mid 3)$ structure. In section 6 , we conclude this paper and further discuss open problems and future directions. In appendix $\mathrm{A}$, we explain an $\mathcal{N}=2$ version of higher rank FZZ-duality based on the results of [22, 23]. In appendix B, we summarize useful results on free field realizations of BP-algebra in [33].

\section{Symmetry algebra}

As explained in the introduction, a class of VOA denoted by $Y_{N_{1}, N_{2}, N_{3}}[\psi]$ was introduced in [9] through brane junctions. There are three types of definitions of the algebra, but the equivalence of the algebras defined in the three ways is still a conjecture if all labels are non-zero. The first one is directly obtained from the brane junction picture, and the symmetry algebra of the coset model (1.3) is identified as $Y_{0, N, N+1}[\psi]$ with

$$
\psi=-k+N+1
$$

after decoupling a $\mathfrak{u}(1)$-sector [9]. The second one is realized by a truncation of a $\mathrm{W}_{1+\infty}[\lambda]$ algebra, which will be explained in the next subsection. The equivalence of these two realizations if one of the three labels is zero has been proven in [4]. The third one is given by an intersection of kernels of screening charges in a free field theory, which is actually the definition of $W_{N_{1}, N_{2}, N_{3}}$ mentioned in the introduction. This screening realization has been proven to coincide with the coset definition in a special series of cases [14]. The algebra $Y_{0, N, N+1}[\psi]$ is dual to $Y_{N, 0, N+1}\left[\psi^{-1}\right][4]$, and the conjectural screening charges for a free field realization of the algebra $Y_{N, 0, N+1}\left[\psi^{-1}\right]$ are provided in subsection 2.2. 


\subsection{Truncation of $\mathrm{W}_{\infty}$-algebra}

In this subsection, we realize the coset algebra by a one-parameter subquotient of the $W_{\infty}[\lambda]$-algebra [4]. Since $\mathfrak{s l}(N)$ with level $k$ may be regarded as $\mathfrak{s u}(N)$ with level $-k,{ }^{3}$ we rewrite the coset (1.3) via level-rank duality as

$$
\frac{\mathfrak{s u}(N+1)_{-k}}{\mathfrak{s u}(N)_{-k} \times \mathfrak{u}(1)} \simeq \frac{\mathfrak{s u}(-k)_{N} \times \mathfrak{s u}(-k)_{1}}{\mathfrak{s u}(-k)_{N+1}}
$$

If $-k=L$ with integer $L \geq 2$, then the symmetry of the coset is known to be the usual $\mathrm{W}_{L}$-algebra. However, we are interested in the parameter regime of $-k<-N-1$ now, thus the symmetry is an "analytic continuation" of the algebra. The extended algebra is a $\mathrm{W}_{\infty}$-algebra called as $\mathrm{W}_{\infty}[\lambda]$ parametrized by $\lambda$ and a central charge $c$. It is rigorously constructed by Andrew Linshaw [41]. For the coset (1.3), $\lambda=-k$ and the central charge is (1.4). It is sometimes convenient to combine a $\mathfrak{u}(1)$-sector to form $\mathrm{W}_{1+\infty}[\lambda]$.

The algebra $\mathrm{W}_{\infty}[\lambda]$ has a spin $s$ current $\mathcal{W}^{(s)}$ with $s=2,3, \ldots$ for generic value of $\lambda$. An ideal forms at $\lambda=L$ with integer $L \geq 2$ and $\mathrm{W}_{L}$-algebra with the truncation of spin as $s=2,3, \ldots L$ can be obtained by dividing the ideal. The operator product expansions (OPEs) among generators are unique up to the two parameters $\lambda, c$ mentioned above. The algebra is known to have a triality relation [41, 42] and it is useful to introduce three parameters $\lambda_{1}, \lambda_{2}, \lambda_{3}$ satisfying [43]

$$
\frac{1}{\lambda_{1}}+\frac{1}{\lambda_{2}}+\frac{1}{\lambda_{3}}=0
$$

One of them is identified with $\lambda$ in $W_{\infty}[\lambda]$ and the central charge is related as

$$
c=\left(\lambda_{1}-1\right)\left(\lambda_{2}-1\right)\left(\lambda_{3}-1\right) .
$$

In order to read off the parameters $\lambda_{j}$ from the OPEs, it is convenient to focus on the normalization independent combination of coefficients given by

$$
\frac{\left(C_{33}^{4}\right)^{2} C_{44}^{0}}{\left(C_{33}^{0}\right)^{2}} .
$$

Here $C_{i j}^{k}$ represents the coefficient of operator product expansion of $\mathcal{W}^{(i)} \mathcal{W}^{(j)}$ in front of $\mathcal{W}^{(k)}$. We further set $\mathcal{W}^{(0)}=\mathbb{1}$. The quantity is related to $\lambda_{i}$ by

$$
\frac{\left(C_{33}^{4}\right)^{2} C_{44}^{0}}{\left(C_{33}^{0}\right)^{2}}=\frac{144(c+2)\left(\lambda_{1}-3\right)\left(\lambda_{2}-3\right)\left(\lambda_{3}-3\right)}{c(5 c+22)\left(\lambda_{1}-2\right)\left(\lambda_{2}-2\right)\left(\lambda_{3}-2\right)} .
$$

In the current case, we may set (see, e.g., (2.19) of [44])

$$
\lambda_{1}=-k, \quad \lambda_{2}=-\frac{k}{k-N-1}, \quad \lambda_{3}=\frac{k}{k-N},
$$

\footnotetext{
${ }^{3}$ For the discussion of vertex operator algebras, the elements of Lie algebras are assumed to take complex values.
} 
which leads to

$$
\begin{aligned}
\frac{\left(C_{33}^{4}\right)^{2} C_{44}^{0}}{\left(C_{33}^{0}\right)^{2}}= & \frac{1}{2 k^{2}(5 N+11)-k(N(5 N+39)+22)+17 N(N+1)} \\
& \times \frac{144(k-1)(k+3)(N+1)(2 k-3 N)(k-N-1)(k-N)(2 k-N)(4 k-3(N+1))}{(k+1)(k+2) N(k-2 N)(2 k-N-1)(3 k-2(N+1))} .
\end{aligned}
$$

We will utilize this to check whether theories have expected symmetries in the analysis below.

Above, we have argued that the algebra $\mathrm{W}_{\infty}[\lambda]$ can be truncated to $\mathrm{W}_{L}$ at $\lambda=L$. More generically, it is known that there is a truncation when $\lambda_{j}$ with $j=1,2,3$ satisfy

$$
\frac{N_{1}}{\lambda_{1}}+\frac{N_{2}}{\lambda_{2}}+\frac{N_{3}}{\lambda_{3}}=1
$$

with non-negative integer $N_{j}[44,45]$. The truncated algebra is supposed to be $Y_{N_{1}, N_{2}, N_{3}}[\psi]$ algebra. For the symmetry algebra of the coset model (1.3), the non-negative integers $N_{j}$ should be set as

$$
N_{1}=0, \quad N_{2}=N, \quad N_{3}=N+1 .
$$

\subsection{Intersection of kernels of screening charges}

Screening charges for free field realization of $Y_{N_{1}, N_{2}, N_{3}}[\psi]$-algebra were proposed in [20] by extending the previous works of $[18,19]$. In this subsection, we explicitly obtain screening charges for $Y_{N, 0, N+1}\left[\psi^{-1}\right]$-algebra by following their construction. There are many equivalent ways to express them but here we use the one resemble to those for $\mathfrak{s l}(N+1 \mid N)$ Toda field theory explained in appendix A.

For $Y_{N_{1}, N_{2}, N_{3}}[\psi]$-algebra, we introduce $\left(N_{1}+N_{2}+N_{3}\right)$ free bosons with labels $\phi_{j}^{(\kappa)}$ $\left(\kappa=1,2,3, j=1,2, \ldots, N_{\kappa}\right)$. The OPEs of the free bosons are normalized as

$$
\phi_{j}^{(\kappa)}(z) \phi_{j^{\prime}}^{\left(\kappa^{\prime}\right)}(0) \sim-\frac{h_{\kappa}}{h_{1} h_{2} h_{3}} \delta^{\kappa, \kappa^{\prime}} \delta_{j, j^{\prime}} \ln z
$$

For our purpose, we set $N_{1}=N, N_{2}=0, N_{3}=N+1$ and (see, e.g., (1.2) of [20])

$$
h_{1}=i \sqrt{k-N-1}, \quad h_{2}=\frac{i}{\sqrt{k-N-1}}, \quad h_{3}=-i \frac{k-N}{\sqrt{k-N-1}} .
$$

The expression of free field realization is determined by the order of free bosons. We choose the one corresponding to the order $\phi_{1}^{(3)} \phi_{1}^{(1)} \phi_{2}^{(3)} \ldots \phi_{N}^{(1)} \phi_{N+1}^{(3)}$. In this case, the screening charges are given by

$$
Q_{l}=\oint d w V_{l}(w)
$$

for $l=1,2, \ldots, 2 N$ with the screening operators as

$$
V_{2 j-1}(w)=e^{-h_{1} \phi_{j}^{(3)}+h_{3} \phi_{j}^{(1)}}, \quad V_{2 j}(w)=e^{-h_{3} \phi_{j}^{(1)}+h_{1} \phi_{j+1}^{(3)}}
$$

for $j=1,2, \ldots, N$. 
Among $(2 N+1)$ free bosons, we decouple a linear combination, $\sum_{j=1}^{N+1} \phi_{j}^{(3)}+\sum_{j=1}^{N} \phi_{j}^{(1)}$, which generates a $\mathfrak{u}(1)$-sector. For this, we redefine the free fields as

$$
\begin{aligned}
\phi_{j} & =-i\left(\phi_{j}^{(3)}-\phi_{j+1}^{(3)}\right) \quad(j=1,2, \ldots, N), \\
\varphi_{j} & =i \sqrt{\frac{k-N}{k-N-1}}\left(\phi_{j}^{(1)}-\phi_{j+1}^{(1)}\right) \quad(j=1,2, \ldots, N-1), \\
\chi & =\sqrt{\frac{N(N+1)}{2 k}}\left(-\frac{h_{1}}{N+1} \sum_{j=1}^{N+1} \phi_{j}^{(3)}+\frac{h_{3}}{N} \sum_{j=1}^{N} \phi_{j}^{(1)}\right) .
\end{aligned}
$$

The OPEs among these fields are

$$
\phi_{i}(z) \phi_{j}(0) \sim-G_{i j}^{(N+1)} \ln z, \quad \varphi_{i}(z) \varphi_{j}(0) \sim G_{i j}^{(N)} \ln z, \quad \chi(z) \chi(0) \sim \frac{1}{2} \ln z,
$$

where $G_{i j}^{(N+1)}$ is the Cartan matrix of $\mathfrak{s l}(N+1)$. We will use its inverse denoted by $G^{(N+1) i j}$ as well. With these new fields, the screening operators are given by

$$
\begin{gathered}
V_{1}=e^{\phi^{1} / b_{(N+1)}-\varphi^{1} / b_{(N)}+\sqrt{\frac{2 k}{N(N+1)}} \chi}, \\
V_{2}=e^{\left(\phi^{1}-\phi^{2}\right) / b_{(N+1)}+\varphi^{1} / b_{(N)}-\sqrt{\frac{2 k}{N(N+1)}} \chi}, \\
V_{3}=e^{\left(\phi^{2}-\phi^{1}\right) / b_{(N+1)}+\left(\varphi^{1}-\varphi^{2}\right) / b_{(N)}+\sqrt{\frac{2 k}{N(N+1)}} \chi}, \\
V_{4}=e^{\left(\phi^{2}-\phi^{3}\right) / b_{(N+1)}+\left(\varphi^{2}-\varphi^{1}\right) / b_{(N)}-\sqrt{\frac{2 k}{N(N+1)}} \chi}, \\
\vdots \\
V_{2 N-1}=e^{\left(\phi^{N}-\phi^{N-1}\right) / b_{(N+1)}+\varphi^{N-1} / b_{(N)}+\sqrt{\frac{2 k}{N(N+1)}} \chi}, \\
V_{2 N}=e^{\phi^{N} / b_{(N+1)}-\varphi^{N-1} / b_{(N)}-\sqrt{\frac{2 k}{N(N+1)}} \chi},
\end{gathered}
$$

where we have set

$$
b_{(a)}=\frac{1}{\sqrt{k-a}}
$$

with $a=N, N+1$. The indices of $\phi_{j}$ and $\varphi_{j}$ are raised as

$$
\phi^{i}=G^{(N+1) i j} \phi_{j}, \quad \varphi^{i}=G^{(N) i j} \varphi_{j} .
$$

In subsequent sections, we shall see that the free field realization corresponds to the theory dual to the coset model (1.3) for explicit examples.

\section{Coset model}

In this section, we examine the coset model (1.3) with focusing on the $N=2$ case given by

$$
\frac{\mathfrak{s l}(3)_{k}}{\mathfrak{s l}(2)_{k} \times \mathfrak{u}(1)}
$$


In the next subsection, we explain the first order formulation of $\mathfrak{s l}(3)$ WZNW model and the free field realization of $\mathfrak{s l}(3)$ current algebra. In subsection 3.2, we obtain an action describing the coset model (3.1) and a free field realization of coset algebra. In subsection 3.3, we explain the spectral flow automorphism of $\mathfrak{s l}(N+1)$ current algebra.

\subsection{First order formulation of WZNW model}

For the proof of FZZ-duality in [26], the reduction method of [27] was utilized, where the analysis relies on a free field realization of $\mathfrak{s l}(2)$ current algebra. This implies that a free field realization of coset algebra is necessary in order to apply the analysis to the current case. In this and next subsections, we review the method developed in [39, 40] and apply it to the coset (3.1).

The symmetry algebra of the coset (1.3) can be constructed from $\mathfrak{s l}(N+1)$ currents by dividing the denominator algebra, $\mathfrak{s l}(N) \times \mathfrak{u}(1)$. The strategy of $[39,40]$ is first expressing the $\mathfrak{s l}(N+1)$ currents by free fields and then reducing the field space such as to be orthogonal to the denominator one. We thus start from a free field realization of $\mathfrak{s l}(3)$ current algebra. We use the first order formulation of $\mathfrak{s l}(3)$ WZNW model corresponding to a free field realization, ${ }^{4}$

$$
\begin{aligned}
S= & \frac{1}{2 \pi} \int d^{2} w\left[\frac{G_{i j}^{(3)}}{2} \partial \phi^{i} \bar{\partial} \phi^{j}+\frac{b_{(3)}}{4} \sqrt{g} \mathcal{R}\left(\phi^{1}+\phi^{2}\right)+\sum_{\alpha=1}^{3}\left(\beta_{\alpha} \bar{\partial} \gamma_{\alpha}+\bar{\beta}_{\alpha} \partial \bar{\gamma}_{\alpha}\right)\right] \\
& -\frac{1}{2 \pi k} \int d^{2} w\left[e^{b_{(3)} \phi_{1}}\left(\beta_{1}-\gamma_{2} \beta_{3}\right)\left(\bar{\beta}_{1}-\bar{\gamma}_{2} \bar{\beta}_{3}\right)+e^{b_{(3)} \phi_{2}} \beta_{2} \bar{\beta}_{2}\right] .
\end{aligned}
$$

Here we set $b_{(3)}=1 / \sqrt{k-3}$ as in (2.18) with $a=3$ and use the Cartan matrix and its inverse expressed as

$$
G_{i j}^{(3)}=\left(\begin{array}{cc}
2 & -1 \\
-1 & 2
\end{array}\right), \quad G^{(3) i j}=\left(\begin{array}{ll}
2 / 3 & 1 / 3 \\
1 / 3 & 2 / 3
\end{array}\right)
$$

The worldsheet metric is denoted as $g_{\mu \nu}$ with $g=\operatorname{det} g_{\mu \nu}$ and the worldsheet curvature as $\mathcal{R}$. In terms of OPEs, the free fields satisfy

$$
\phi_{i}(z) \phi_{j}(0) \sim-G_{i j}^{(3)} \ln |z|^{2}, \quad \gamma_{\alpha}(z) \beta_{\alpha^{\prime}}(0) \sim \frac{\delta_{\alpha, \alpha^{\prime}}}{z} .
$$

The generators of the $\mathfrak{s l}(3)$ current algebra can be written in terms of the free fields as (see, e.g., [47])

$$
\begin{aligned}
& e^{1}=\beta_{1}, \quad e^{2}=\beta_{2}-\gamma_{1} \beta_{3}, \quad e^{3}=\beta_{3}, \\
& h^{1}=b_{(3)}^{-1} \partial \phi_{1}+2 \gamma_{1} \beta_{1}-\gamma_{2} \beta_{2}+\gamma_{3} \beta_{3}, \\
& h^{2}=b_{(3)}^{-1} \partial \phi_{2}-\gamma_{1} \beta_{1}+2 \gamma_{2} \beta_{2}+\gamma_{3} \beta_{3} .
\end{aligned}
$$

\footnotetext{
${ }^{4}$ The action can be derived from a particular parametrization of group element as in (2.7) of [32]. Here, $\phi_{1}, \phi_{2}$ takes real values, while $\gamma_{\alpha}$ and $\bar{\gamma}_{\alpha}$ are related by complex conjugations. See, e.g., [46] for the analogues case of $\mathfrak{s l}(2)$ in [27].
} 
Here and in the following, the normal ordering prescription is assumed for the products of fields. The other generators $f^{1}, f^{2}, f^{3}$ can be expressed in a similar manner. The energymomentum tensor is given by

$$
T=-\frac{1}{2} G_{i j}^{(3)} \partial \phi^{i} \partial \phi^{j}+b_{(3)} \partial^{2}\left(\phi^{1}+\phi^{2}\right)-\sum_{\alpha=1}^{3} \partial \gamma_{\alpha} \beta_{\alpha} .
$$

\subsection{First order formulation of coset model}

Following [39, 40], we perform a projection of the numerator algebra, $\mathfrak{s l}(3)$, into a subsector orthogonal to the denominator algebra, $\mathfrak{s l}(2) \times \mathfrak{u}(1)$. The generators of $\mathfrak{s l}(2)$ and $\mathfrak{u}(1)$ current algebras may be denoted as $\{\hat{h}, \hat{e}, \hat{f}\}$ and $\hat{h}^{\prime}$. The Cartan subalgebras are generated by

$$
\hat{h}=h^{1}+h^{2}, \quad \hat{h}^{\prime}=h^{1}-h^{2} .
$$

For the generators of $\mathfrak{s l}(2)$, we choose $\hat{e}=e^{3}$, which determines $\hat{f}$ as well. ${ }^{5}$ We introduce two free bosons by

$$
\hat{h}=2 b_{(2)}^{-1} \partial \hat{\varphi}+2 \gamma_{3} \beta_{3}, \quad \hat{h}^{\prime}=2 \sqrt{3 k} \partial \hat{\chi},
$$

where $b_{(2)}=1 / \sqrt{k-2}$ as in (2.18) with $a=2$. This implies that the free bosons satisfy

$$
\partial \hat{\varphi}(z) \partial \hat{\varphi}(0) \sim-\frac{1}{2 z^{2}}, \quad \partial \hat{\chi}(z) \partial \hat{\chi}(0) \sim-\frac{1}{2 z^{2}}
$$

We define the coset theory by considering the subsector orthogonal to $\hat{\varphi}, \hat{\chi}, \gamma_{3}, \beta_{3}, \bar{\gamma}_{3}, \bar{\beta}_{3}$. For the ghost part with $\gamma_{3}, \beta_{3}, \bar{\gamma}_{3}, \bar{\beta}_{3}$, we simply decouple it from the rest.

Let us check that the first order formulation of the coset theory possesses the correct symmetry algebra. The free fields are $\phi_{1}, \phi_{2}$ and the ghost systems $\left(\gamma_{j}, \beta_{j}\right)$ with $j=$ 1,2 . We require that the symmetry currents do not have non-trivial OPEs with $\hat{\varphi}, \hat{\chi}$ and commute with the interaction terms

$$
S_{1}=\int d^{2} w e^{b_{(3)} \phi_{1}} \beta_{1} \bar{\beta}_{1}, \quad S_{2}=\int d^{2} w e^{b_{(3)} \phi_{2}} \beta_{2} \bar{\beta}_{2} .
$$

There are no spin-one currents satisfying these conditions. We find a spin-two current, which is the energy-momentum tensor,

$$
\begin{aligned}
T= & -\frac{1}{2} G_{i j}^{(3)} \partial \phi^{i} \partial \phi^{j}+b_{(3)} \partial^{2}\left(\phi^{1}+\phi^{2}\right)-\sum_{i=1}^{2} \partial \gamma_{i} \beta_{i} \\
& +\partial \hat{\varphi} \partial \hat{\varphi}-b_{(2)} \partial^{2} \hat{\varphi}+\partial \hat{\chi} \partial \hat{\chi} .
\end{aligned}
$$

Its central charge reproduces (1.4) with $N=2$. In a similar way, we find a spin-three current and a spin-four current. The ratio of OPE coefficients in (2.6) reproduces (2.8) with $N=2$.

\footnotetext{
${ }^{5}$ This choice is different from that of standard coset construction in [48], but the algebras obtained in the two ways are argued to be isomorphic with each other $[39,40]$. In the current case, the algebra of the coset reproduces the expected one as will be checked below.
} 
We shall examine the correlation functions of the coset theory. The vertex operators of the $\mathfrak{s l}(3)$ WZNW model are expressed in the form

$$
V(z)=P\left(\gamma_{\alpha}, \bar{\gamma}_{\alpha}\right) e^{b_{(3)}\left(j_{1} \phi^{1}+j_{2} \phi^{2}\right)},
$$

where $P\left(\gamma_{\alpha}\right)$ is a function of $\gamma_{\alpha}, \bar{\gamma}_{\alpha}$ with $\alpha=1,2,3$. In the coset theory, we consider a subsector orthogonal to $\hat{\varphi}, \hat{\chi}$ and decouple $\gamma_{3}, \beta_{3}, \bar{\gamma}_{3}, \bar{\beta}_{3}$. We then introduce counter parts $\varphi, \chi$ with negative kinetic terms so as to cancel the contributions from $\hat{\varphi}, \hat{\chi}^{6}{ }^{6}$ The action of the coset theory is now

$$
\begin{aligned}
S= & \frac{1}{2 \pi} \int d^{2} w\left[\frac{G_{i j}^{(3)}}{2} \partial \phi^{i} \bar{\partial} \phi^{j}-\partial \varphi \bar{\partial} \varphi-\partial \chi \partial \chi+\frac{1}{4} \sqrt{g} \mathcal{R}\left(b_{(3)}\left(\phi^{1}+\phi^{2}\right)-b_{(2)} \varphi\right)\right] \\
& +\frac{1}{2 \pi} \int d^{2} w\left[\sum_{i=1}^{2}\left(\beta_{i} \bar{\partial} \gamma_{i}+\bar{\beta}_{i} \partial \bar{\gamma}_{i}\right)-\frac{1}{k}\left(e^{b_{(3)} \phi_{1}} \beta_{1} \bar{\beta}_{1}+e^{b_{(3)} \phi_{2}} \beta_{2} \bar{\beta}_{2}\right)\right] .
\end{aligned}
$$

We consider vertex operators of the form

$$
\Psi(z)=\gamma_{1}^{-j_{1}-l-m} \gamma_{2}^{-j_{2}-l+m} \bar{\gamma}_{1}^{-j_{1}-l-\bar{m}} \bar{\gamma}_{2}^{-j_{2}-l+\bar{m}} e^{b_{(3)}\left(j_{1} \phi_{1}+j_{2} \phi_{2}\right)} e^{2 b_{(2)} l \varphi+2 \sqrt{\frac{3}{k}}\left(m \chi_{L}+\bar{m} \chi_{R}\right)} .
$$

Here we have decomposed as $\chi(z, \bar{z})=\chi_{L}+\chi_{R} \cdot{ }^{7}$

\subsection{Spectral flow}

As in [26], we need to include the effects of spectral flow automorphism of $\mathfrak{s l}(N+1)$ current algebra. We are using the notation such that commutation relations among mode expansions of generators are given by

$$
\begin{array}{ll}
{\left[h_{n}^{i}, h_{m}^{j}\right]=-2 k n \delta_{i, j} \delta_{n+m, 0},} & {\left[h_{n}^{i}, e_{m}^{j}\right]=G_{i j}^{(N+1)} e_{n+m}^{j},} \\
{\left[h_{n}^{i}, f_{m}^{j}\right]=-G_{i j}^{(N+1)} f_{n+m}^{j},} & {\left[e_{n}^{i}, f_{m}^{j}\right]=-\delta_{i, j} h_{n+m}^{i}+k n \delta_{i, j} \delta_{n+m, 0} .}
\end{array}
$$

The same commutation relations are satisfied even after the changes

$$
\rho^{\left\{S_{j}\right\}}\left(e_{n}^{i}\right)=e_{n-S_{i}}^{i}, \quad \rho^{\left\{S_{j}\right\}}\left(f_{n}^{i}\right)=f_{n+S_{i}}^{i}, \quad \rho^{\left\{S_{j}\right\}}\left(h_{n}^{i}\right)=h_{n}^{i}+k S_{i} \delta_{n, 0}
$$

for $i=1,2, \ldots, N$. The commutation relations among new generators fix the action of $\rho^{\left\{S_{j}\right\}}$ to the other generators. From this, we can see that $\mathfrak{s l}(N+1)$ current algebra possesses $N$-parameter family of spectral flow automorphism.

\footnotetext{
${ }^{6}$ The coset $\mathfrak{s l}(2) / \mathfrak{u}(1)$ can be obtained by the decomposition $\mathfrak{s l}(2) \subset \mathfrak{s l}(2) / \mathfrak{u}(1) \times \mathfrak{u}(1)$. This implies that the correlator of the coset is obtained from that of $\mathfrak{s l}(2)$ WZNW model divided by that of $\mathfrak{u}(1)$ theory. The inverse of correlator in the $\mathfrak{u}(1)$ theory can be computed with the opposite sign in the kinetic term. The same technique can be applied directly for the theory of $\chi$. It seems natural to expect that the same is true for the theory of $\varphi$, but this is something to be confirmed.

${ }^{7}$ We may define the dual field $\tilde{\chi}(z, \bar{z})$ via $\tilde{\chi}=-i \int^{z, \bar{z}} * d \chi$, where $* d \chi$ denotes the Hodge dual of the differential $d \chi$. Then the decomposition is such that $\chi(z, \bar{z})=\chi_{L}(z, \bar{z})+\chi_{R}(z, \bar{z})$ and $\tilde{\chi}(z, \bar{z})=\chi_{L}(z, \bar{z})-$ $\chi_{R}(z, \bar{z})$. See, e.g., [26].
} 
In the rest of this subsection, we focus on the $N=2$ case and express $\rho^{S_{1}, S_{2}} \equiv \rho^{\left\{S_{j}\right\}}$. We define a state $\left|S_{1}, S_{2}\right\rangle$ satisfying

$$
\rho^{S_{1}, S_{2}}\left(e_{n}^{\alpha}\right)\left|S_{1}, S_{2}\right\rangle=0, \quad \rho^{S_{1}, S_{2}}\left(f_{n}^{\alpha}\right)\left|S_{1}, S_{2}\right\rangle=0, \quad \rho^{S_{1}, S_{2}}\left(h_{n}^{i}\right)\left|S_{1}, S_{2}\right\rangle=0
$$

with $\alpha=1,2,3, i=1,2$ and $n=0,1,2, \ldots$. We may decompose the state in terms of free fields as

$$
\left|S_{1}, S_{2}\right\rangle=\left|S_{1}, S_{2}\right\rangle_{(\beta, \gamma)} \otimes\left|S_{1}, S_{2}\right\rangle_{\phi}
$$

Here we require

$$
\begin{aligned}
& \beta_{1, n-S_{1}}\left|S_{1}, S_{2}\right\rangle_{(\beta, \gamma)}=\beta_{2, n-S_{2}}\left|S_{1}, S_{2}\right\rangle_{(\beta, \gamma)}=\beta_{3, n-S_{1}-S_{2}}\left|S_{1}, S_{2}\right\rangle_{(\beta, \gamma)}=0, \\
& \gamma_{1, n+S_{1}}\left|S_{1}, S_{2}\right\rangle_{(\beta, \gamma)}=\gamma_{2, n+S_{2}}\left|S_{1}, S_{2}\right\rangle_{(\beta, \gamma)}=\gamma_{3, n+S_{1}+S_{2}}\left|S_{1}, S_{2}\right\rangle_{(\beta, \gamma)}=0
\end{aligned}
$$

for $n=0,1,2, \ldots$ and

$$
\left|S_{1}, S_{2}\right\rangle_{\phi}=e^{\left(S_{1} \phi^{1}(0)+S_{2} \phi^{2}(0)\right) / b_{(3)}}|0\rangle_{\phi} .
$$

We denote the corresponding operator as $v^{S_{1}, S_{2}}(\xi)$ inserted at $w=\xi$. The insertion of this operator puts a restriction on the domain of integration over $\beta_{1}, \beta_{2}, \beta_{3}$ such that they have zeros of order $S_{1}, S_{2}, S_{1}+S_{2}$, respectively. Moreover, it also induces the insertion of $e^{\left(S_{1} \phi^{1}+S_{2} \phi^{2}\right) / b_{(3)}}(\xi)$. See [26] for more details in the $N=1$ case. In the following, we assume that $S_{1}, S_{2} \geq 0$, which may be realized by utilizing the Weyl symmetry of $\mathfrak{s l}(3)$ Lie algebra when only one of the vertex operators inserted has non-zero $S_{1}, S_{2}$ as in (4.4) below.

\section{A generalized FZZ-duality}

Now that we have a first order formulation of the coset model (3.1), we can apply the method of $[26,27]$ to derive a duality. We review the analysis of [33] on the reduction from $\mathfrak{s l}(3)$ WZNW model to $\mathfrak{s l}(3)$ Toda field theory in the next subsection, and we apply the reduction procedure to the coset theory in subsection 4.2. We rewrite the correlators such that they can be interpreted as those of the dual theory with an $\mathfrak{s l}(3 \mid 2)$ structure in subsection 4.3 .

\subsection{Reduction from WZNW model}

In [33], we have explored correspondences of theories with different $\mathrm{W}$-algebra symmetry. In this subsection, we review a result on the reduction from $\mathfrak{s l}(3)$ WZNW model to $\mathfrak{s l}(3)$ Toda field theory. The relation of correlation functions obtained by the reduction procedure is generically complicated. However, as shown in [33], a simplification occurs if we consider a restricted setup where vertex operators are of specific from. In order to obtain the first order formulation realization of the coset theory, we decouple a ghost system $\left(\gamma_{3}, \beta_{3}\right)$, and this is exactly the condition where the simplification occurs.

In our construction of coset theory, we use the vertex operators of the form (3.14). It is convenient to rewrite them as

$$
\Psi_{\nu}\left(z_{\nu}\right)=\Phi_{\nu}\left(z_{\nu}\right) V_{l^{\nu} ; m^{\nu}, \bar{m}^{\nu}}^{\varphi, \chi}\left(z_{\nu}\right), \quad V_{l^{\nu} ; m^{\nu}, \bar{m}^{\nu}}^{\varphi, \chi}\left(z_{\nu}\right)=e^{2 b_{(2)} l^{\nu} \varphi+2 \sqrt{\frac{3}{k}}\left(m^{\nu} \chi_{L}+\bar{m}^{\nu} \chi_{R}\right)},
$$


where $\Phi_{\nu}\left(z_{\nu}\right)$ is given in terms of a Mellin transform as

$$
\begin{aligned}
& \Phi_{\nu}\left(z_{\nu}\right)=\int \frac{d^{2} \mu_{1}^{\nu} d^{2} \mu_{2}^{\nu}}{\left|\mu_{1}^{\nu}\right|^{2}\left|\mu_{2}^{\nu}\right|^{2}}\left(\mu_{1}^{\nu}\right)^{l^{\nu}+m^{\nu}}\left(\bar{\mu}_{1}^{\nu}\right)^{l^{\nu}+\bar{m}^{\nu}}\left(\mu_{2}^{\nu}\right)^{l^{\nu}-m^{\nu}}\left(\bar{\mu}_{2}^{\nu}\right)^{l^{\nu}-\bar{m}^{\nu}} V_{\nu}\left(z_{\nu}\right), \\
& V_{\nu}\left(z_{\nu}\right)=\left|\mu_{1}\right|^{2 j_{1}^{\nu}}\left|\mu_{2}\right|^{2 j_{2}^{\nu}} e^{\mu_{1}^{\nu} \gamma_{1}+\mu_{2}^{\nu} \gamma_{2}-\bar{\mu}_{1}^{\nu} \bar{\gamma}_{1}-\bar{\mu}_{2}^{\nu} \bar{\gamma}_{2}} e^{b_{(3)}\left(j_{1}^{\nu} \phi_{1}+j_{2}^{\nu} \phi_{2}\right)} .
\end{aligned}
$$

The pre-factor $\left|\mu_{1}\right|^{2 j_{1}^{\nu}}\left|\mu_{2}\right|^{2 j_{2}^{\nu}}$ in $V_{\nu}\left(z_{\nu}\right)$ is chosen such that the expressions obtained later become simpler. We examine the correlation functions of $\mathfrak{s l}(3)$ WZNW model and apply the results to the case of coset theory in next subsection.

The correlation functions of $\mathfrak{s l}(3)$ WZNW model can be written in the path integral formulation as

$$
\begin{aligned}
& \left\langle\prod_{\nu=1}^{N} V_{\nu}\left(z_{\nu}\right) v^{S_{1}, S_{2}}(\xi)\right\rangle \\
& \quad=\int_{S_{j}, \xi} \mathcal{D} \phi_{1} \mathcal{D} \phi_{2}\left[\prod_{\alpha=1}^{3} \mathcal{D}^{2} \beta_{\alpha} \mathcal{D}^{2} \gamma_{\alpha}\right] e^{-S} \prod_{\nu=1}^{N} V_{\nu}\left(z_{\nu}\right) e^{\left(S_{1} \phi^{1}+S_{2} \phi^{2}\right) / b_{(3)}}(\xi) .
\end{aligned}
$$

As in the case of the original FZZ-duality in [26], we consider the correlation functions with the insertion of $v^{S_{1}, S_{2}}(\xi)$ in order to deal with a generic type of coset correlators. ${ }^{8}$ The domain of integration for $\beta_{\alpha}, \bar{\beta}_{\alpha}$ is then restricted as described above. Here the action is (3.2) and the vertex operators are of the form (4.3). Since the vertex operators do not depend on $\gamma_{3}, \bar{\gamma}_{3}$, we can integrate out $\gamma_{3}, \beta_{3}, \bar{\gamma}_{3}, \bar{\beta}_{3}$. Then, we can simply set $\beta_{3}=\bar{\beta}_{3}=0$, and the action reduces to a part appearing in (3.13).

With this setup, we can integrate out $\gamma_{1}, \gamma_{2}, \bar{\gamma}_{1}, \bar{\gamma}_{2}$. This results in the constraints

$$
\bar{\partial} \beta_{i}(w)=-2 \pi \sum_{\nu=1}^{N} \mu_{i}^{\nu} \delta^{(2)}\left(w-z_{\nu}\right), \quad \partial \bar{\beta}_{i}(w)=2 \pi \sum_{\nu=1}^{N} \bar{\mu}_{i}^{\nu} \delta^{(2)}\left(w-z_{\nu}\right)
$$

with $i=1,2$. Solving the equations, we have

$$
\beta_{i}(w)=-\sum_{\nu=1}^{N} \frac{\mu_{i}^{\nu}}{w-z_{\nu}}
$$

and similarly for $\bar{\beta}_{i}$. Because of the insertion of $v^{S_{1}, S_{2}}(\xi), \beta_{i}$ should have a zero of order $S_{i}$ at $w=\xi$. This leads to the constraints

$$
\sum_{\nu=1}^{N} \frac{\mu_{i}^{\nu}}{\left(\xi-z_{\nu}\right)^{n}}=0
$$

\footnotetext{
${ }^{8}$ In the correlation function of $\mathfrak{s l}(2)_{k} / \mathfrak{u}(1)$ coset, the total $\mathfrak{u}(1)$-charges are known to be violated up to $\left|\sum_{\nu} m_{\nu}\right|=\left|\sum_{\nu} \bar{m}_{\nu}\right|=\frac{k}{2} S \leq \frac{k}{2}(N-2)$, see, e.g., [49]. In order to realize this violation, we need to insert an identity operator $\mathbb{1}=v^{S}(\xi) e^{-\sqrt{k} S \chi}$ as explained in [26]. Since we can construct analogues identity operators including $v^{S_{1}, S_{2}}(\xi)$ for the current coset as in (4.14) below, the generic type of coset correlator should include the insertion of identity operator with some $S_{1}, S_{2}$.
} 
for $n=0,1, \ldots, S_{i}$. Noticing that a holomorphic one-form with $N$ poles should have $(N-2)$ zeros on a Riemann sphere, we can rewrite (4.6) as ${ }^{9}$

$$
\beta_{i}(w)=-u_{i} \frac{(w-\xi)^{S_{i}} \prod_{p=1}^{N-2-S_{i}}\left(w-y_{p}^{i}\right)}{\prod_{\nu=1}^{N}\left(w-z_{\nu}\right)} \equiv-u_{i} \mathcal{B}_{i}\left(w ; z_{\nu}, y_{p}^{i}\right) .
$$

This equation defines the transformation of variables from $\mu_{i}^{\nu}$ to $y_{p}^{i}$.

Inserting (4.8) into the action (3.2) with $\beta_{3}=0$, the coefficients of interaction terms have now coordinate dependence. We remove the coordinate dependence by shifting $\phi_{i}$ as

$$
\phi_{i}+\frac{1}{b_{(3)}} \ln \left|u_{i} \mathcal{B}_{i}\right|^{2} \rightarrow \phi_{i}
$$

with $i=1,2$. The shifts yield several contributions from the kinetic terms of $\phi_{i}$ among others, and the correlation function (4.4) becomes of the form

$$
\begin{aligned}
& \left\langle\prod_{\nu=1}^{N} V_{\nu}\left(z_{\nu}\right) v^{S_{1}, S_{2}}(\xi)\right\rangle \\
& \quad=\left|\Theta_{N}\right|^{2} \prod_{i=1}^{2} \prod_{n=0}^{S_{i}} \delta^{(2)}\left(\sum_{\nu=1}^{N} \frac{\mu_{i}^{\nu}}{\left(\xi-z_{\nu}\right)^{n}}\right)\left\langle\prod_{\nu=1}^{N} \tilde{V}_{\nu}\left(z_{\nu}\right) \prod_{p=1}^{N-2-S_{1}} \tilde{V}_{b}\left(y_{p}^{1}\right) \prod_{p=1}^{N-2-S_{2}} \tilde{V}_{b}\left(y_{p}^{2}\right)\right\rangle .
\end{aligned}
$$

The right hand side is computed with the action of $\mathfrak{s l}(3)$ Toda field theory,

$$
S=\frac{1}{2 \pi} \int d^{2} w\left[\frac{G_{i j}^{(3)}}{2} \partial \phi^{i} \bar{\partial} \phi^{j}+\frac{1}{4} \sqrt{g} \mathcal{R}\left(b_{(3)}+b_{(3)}^{-1}\right)\left(\phi^{1}+\phi^{2}\right)+\frac{1}{k}\left(e^{b_{(3)} \phi_{1}}+e^{b_{(3)} \phi_{2}}\right)\right] .
$$

Later we use the self-duality of $\mathfrak{s l}(3)$ Toda field theory under the exchange of $b_{(3)} \leftrightarrow b_{(3)}^{-1}$. The modified vertex operators are

$$
\tilde{V}_{\nu}\left(z_{\nu}\right)=e^{b_{(3)}\left(j_{1}^{\nu}+b_{(3)}^{-2}\right) \phi_{1}+b_{(3)}\left(j_{2}^{\nu}+b_{(3)}^{-2}\right) \phi_{2}}, \quad \tilde{V}_{b}\left(y_{p}^{i}\right)=e^{-\phi^{i} / b_{(3)}} .
$$

The pre-factor is computed as

$$
\begin{aligned}
\Theta_{N}= & u_{1}{ }^{2-\frac{2 S_{1}+S_{2}}{3 b_{(3)}^{2}}} u_{2} u^{2-\frac{S_{1}+2 S_{2}}{3 b_{(3)}^{2}}} \\
& \times\left[\prod_{\nu<\nu^{\prime}} z_{\nu \nu^{\prime}}\right]^{\frac{2}{b_{(3)}^{2}}}\left[\prod_{\nu, p, i}\left(z_{\nu}-y_{p}^{i}\right)\right]^{-\frac{1}{b_{(3)}^{2}}}\left[\prod_{p, p^{\prime}}\left(y_{p}^{1}-y_{p^{\prime}}^{2}\right)\right]^{\frac{1}{3 b_{(3)}^{2}}}\left[\prod_{p<p^{\prime}, i} y_{p p^{\prime}}^{i}\right]^{\frac{2}{3 b_{(3)}^{2}}},
\end{aligned}
$$

where $z_{\nu \nu^{\prime}}=z_{\nu}-z_{\nu^{\prime}}$ and $y_{p p^{\prime}}^{i}=y_{p}^{i}-y_{p^{\prime}}^{i}$. See $[26,27]$ for more details on the reduction procedure in the case of $\mathfrak{s l}(2)$ WZNW model.

\footnotetext{
${ }^{9}$ In order for the equations (4.8) to make sense, we may need to set $0 \leq S_{1}, S_{2} \leq N-2$. There is a similar bound, $0 \leq S \leq N-2$, for the $\mathfrak{s l}(2)$ case, and this is related to the bound of $\mathfrak{u}(1)$-charge violation discussed in footnote 8 . We expect that similar bounds exist even in the current case, but we cannot say anything concrete without further investigations.
} 


\subsection{Reduction from coset theory}

We apply the reduction relation from $\mathfrak{s l}(3)$ WZNW model to the case with the coset theory by following the analysis of [26]. In order to do so, we need to add free bosons $\varphi, \chi$ and use the vertex operators (4.1) with (4.2) and (4.3). In the coset theory, we may insert an identity operator ${ }^{10}$

$$
\mathbb{1}=v^{S_{1}, S_{2}}(\xi) e^{-\left(S_{1}+S_{2}\right) \varphi / b_{(2)}}(\xi) e^{-\sqrt{\frac{k}{3}}\left(S_{1}-S_{2}\right)\left(\chi_{L}+\chi_{R}\right)}(\xi),
$$

which uses the spectral flow operator $v^{S_{1}, S_{2}}(\xi)$ as mentioned above.

Here we perform the reduction procedure in the previous subsection, where the parameters $\mu_{i}^{\nu}, \bar{\mu}_{i}^{\nu}$ are replaced by $y_{p}^{i}, \bar{y}_{p}^{i}$ with $i=1,2$. The Jacobian due to the change of variables is $[26,28,29]$

$$
\begin{aligned}
& \prod_{\nu=1}^{N} \frac{d^{2} \mu_{i}^{\nu}}{\left|\mu_{i}^{\nu}\right|^{2}} \prod_{n=0}^{S_{i}} \delta^{(2)}\left(\sum_{\nu=1}^{N} \frac{\mu_{i}^{\nu}}{\left(\xi-z_{\nu}\right)^{n}}\right) \\
& \quad=\frac{\prod_{\nu<\nu^{\prime}}^{N}\left|z_{\nu \nu^{\prime}}\right|^{2} \prod_{p<p^{\prime}}^{N-2-S_{i}}\left|y_{p p^{\prime}}^{i}\right|^{2}}{\prod_{\nu=1}^{N} \prod_{p=1}^{N-2-S_{i}}\left|z_{\nu}-y_{p}^{i}\right|^{2}} \frac{d^{2} u_{i}}{\left|u_{i}\right|^{4+2 S_{i}}} \prod_{p=1}^{N-2-S_{i}} d^{2} y_{p}^{i}
\end{aligned}
$$

for each $i=1,2$. Because of the transformation of (4.2), the pre-factors with $u_{i} \mathcal{B}_{i}, \bar{u}_{i} \overline{\mathcal{B}}_{i}$ remain. In order to remove them, we further shift $\varphi, \chi$ as

$$
\begin{aligned}
\varphi+\frac{1}{2 b_{(2)}}\left[\ln \left|u_{1} \mathcal{B}_{1}\right|^{2}+\ln \left|u_{2} \mathcal{B}_{2}\right|^{2}\right] & \rightarrow \varphi, \\
\chi_{L}+\frac{1}{2} \sqrt{\frac{k}{3}}\left[\ln \left(u_{1} \mathcal{B}_{1}\right)-\ln \left(u_{2} \mathcal{B}_{2}\right)\right] & \rightarrow \chi_{L}, \\
\chi_{R}+\frac{1}{2} \sqrt{\frac{k}{3}}\left[\ln \left(\bar{u}_{1} \overline{\mathcal{B}}_{1}\right)-\ln \left(\bar{u}_{2} \overline{\mathcal{B}}_{2}\right)\right] & \rightarrow \chi_{R} .
\end{aligned}
$$

The coset correlation function is now written as

$$
\begin{aligned}
& \left\langle\prod_{\nu=1}^{N} \Psi_{\nu}\left(z_{\nu}\right)\right\rangle=\int \prod_{i=1}^{2} \frac{\prod_{p=1}^{N-2-S_{i}} d^{2} y_{p}^{i}}{\left(N-2-S_{i}\right) !} \\
& \times\left\langle\prod_{\nu=1}^{N} \tilde{V}_{\nu}\left(z_{\nu}\right) V_{l^{\nu}-b_{(2)}^{-1} ; m^{\nu}, \bar{m}^{\nu}}^{\varphi, \chi}\left(z_{\nu}\right) \prod_{p=1}^{N-2-S_{1}} \tilde{V}_{b}\left(y_{p}^{1}\right) V_{\frac{1}{2 b_{(2)}^{2}} ; \frac{k}{6}, \frac{k}{6}}^{\varphi, \chi}\left(y_{p}^{1}\right) \prod_{p=1}^{N-2-S_{2}} \tilde{V}_{b}\left(y_{p}^{2}\right) V_{\frac{1}{2 b_{(2)}^{2}} ;-\frac{k}{6},-\frac{k}{6}}^{\varphi, \chi}\left(y_{p}^{2}\right)\right\rangle .
\end{aligned}
$$

The right hand side is evaluated by the action

$$
\begin{aligned}
S= & \frac{1}{2 \pi} \int d^{2} w\left[\frac{G_{i j}^{(3)}}{2} \partial \phi^{i} \bar{\partial} \phi^{j}-\partial \varphi \bar{\partial} \varphi-\partial \chi \partial \chi+\frac{1}{4} \sqrt{g} \mathcal{R}\left(Q_{\phi}\left(\phi^{1}+\phi^{2}\right)-Q_{\varphi} \varphi\right)\right] \\
& +\frac{1}{k} \int d^{2} w\left[e^{b_{(3)} \phi_{1}}+e^{b_{(3)} \phi_{2}}\right] .
\end{aligned}
$$

\footnotetext{
${ }^{10} \mathrm{As}$ argued in footnote 8 , we may insert an identity operator $\mathbb{1}=v^{S}(\xi) e^{-\sqrt{k} S \chi}$ in the correlation functions of $\mathfrak{s l}(2) / \mathfrak{u}(1)$ coset. In terms of the decomposition $\mathfrak{s l}(2) \subset \mathfrak{s l}(2) / \mathfrak{u}(1) \times \mathfrak{u}(1)$, the spectral flow action to the $\mathfrak{s l}(2)$ part can be reproduced from the corresponding action only to the Cartan part, i.e., the $\mathfrak{u}(1)$ part, see, e.g., [50]. We can obtain $\mathbb{1}=v^{S}(\xi) e^{-\sqrt{k} S \chi}$ by considering the identity operator $\mathbb{1}$ for the $\mathfrak{s l}(2) / \mathfrak{u}(1)$ part and taking the "inverse" of the $\mathfrak{u}(1)$ part. The identity operator in (4.14) can constructed in a similar manner from the decomposition $\mathfrak{s l}(3) \subset \mathfrak{s l}(3) /(\mathfrak{s l}(2) \times \mathfrak{u}(1)) \times \mathfrak{s l}(2) \times \mathfrak{u}(1)$.
} 
The background charges are

$$
Q_{\phi}=b_{(3)}+\frac{1}{b_{(3)}}, \quad Q_{\varphi}=b_{(2)}+\frac{2}{b_{(2)}} .
$$

The division by $\left(N-2-S_{i}\right)$ ! comes from $\left(N-2-S_{i}\right)$ !-fold map between $\mu_{i}^{\nu}$ and $y_{p}^{i}$, see [26] as well. Moreover, note that the pre-factor $\Theta_{N}$ is canceled with similar factors generated due to the shifts (4.16).

\subsection{Dual theory}

In the previous subsection, we have expressed the $N$-point function of the coset model (3.1) as in the right hand side of (4.17). In this subsection, we rewrite the correlation function such as to be the $N$-point function of the theory dual to the coset model (3.1) by following [26].

Firstly, we utilize the self-duality of $\mathfrak{s l}(3)$ Toda field theory, which is an essential point to obtain a strong/weak duality. Instead of (4.18), we use ${ }^{11}$

$$
\begin{aligned}
S= & \frac{1}{2 \pi} \int d^{2} w\left[\frac{G_{i j}^{(3)}}{2} \partial \phi^{i} \bar{\partial} \phi^{j}-\partial \varphi \bar{\partial} \varphi-\partial \chi \partial \chi+\frac{1}{4} \sqrt{g} \mathcal{R}\left(Q_{\phi}\left(\phi^{1}+\phi^{2}\right)-Q_{\varphi} \varphi\right)\right] \\
& +\lambda \int d^{2} w\left[e^{\phi_{1} / b_{(3)}}+e^{\phi_{2} / b_{(3)}}\right]
\end{aligned}
$$

where $b_{(3)}$ in the interaction terms is replaced by $1 / b_{(3)}$.

Secondly, we treat the vertex operators inserted at $w=y_{p}^{i}$ as interaction terms. Namely, we express the correlation function as

$$
\left\langle\prod_{\nu=1}^{N} \Psi_{\nu}\left(z_{\nu}\right)\right\rangle=\left\langle\prod_{\nu=1}^{N} \mathcal{V}_{\nu}\left(z_{\nu}\right)\right\rangle, \quad \mathcal{V}_{\nu}\left(z_{\nu}\right)=\tilde{V}_{\nu}\left(z_{\nu}\right) V_{l^{\nu}-b_{(2)}^{-2} ; m^{\nu}, \bar{m}^{\nu}}^{\varphi, \chi}\left(z_{\nu}\right) .
$$

The action for the right hand side is now

$$
\begin{aligned}
S= & \frac{1}{2 \pi} \int d^{2} w\left[\frac{G_{i j}^{(3)}}{2} \partial \phi^{i} \bar{\partial} \phi^{j}-\partial \varphi \bar{\partial} \varphi-\partial \chi \partial \chi+\frac{1}{4} \sqrt{g} \mathcal{R}\left(Q_{\phi}\left(\phi^{1}+\phi^{2}\right)-Q_{\varphi} \varphi\right)\right] \\
& +\lambda \int d^{2} w \sum_{i=1}^{4} V_{i}
\end{aligned}
$$

with four interaction terms

$$
\begin{array}{lll}
V_{1}=e^{\phi_{1} / b_{(3)}}, & V_{2}=e^{\phi_{2} / b_{(3)}}, \\
V_{3}=e^{-\phi^{1} / b_{(3)}+\varphi / b_{(2)}+\sqrt{\frac{k}{3}} \chi}, & V_{4}=e^{-\phi^{2} / b_{(3)}+\varphi / b_{(2)}-\sqrt{\frac{k}{3}} \chi} .
\end{array}
$$

In this way, we can relate the $N$-point function of the coset model (3.1) with the $N$-point function of a theory with the action (4.22).

\footnotetext{
${ }^{11}$ The coefficient $\lambda$ in front of the interaction terms can be set arbitrary by shifting $\phi_{i}$. This also shifts the overall normalization of vertex operators, which are neglected thorough this paper. Other coefficients in interaction terms appearing below will be set in similar ways.
} 
Finally, we rewrite the action (4.22) such that the interaction terms (4.23) become the same as the screening charges in (2.17). For this, we change the fields as

$$
\begin{aligned}
(k-3) \phi_{1}+(k-2) \phi_{2}-\frac{2}{b_{(2)} b_{(3)}} \varphi & \rightarrow \phi_{1}, \\
(k-2) \phi_{1}+(k-3) \phi_{2}-\frac{2}{b_{(2)} b_{(3)}} \varphi & \rightarrow \phi_{2}, \\
\frac{1}{b_{(2)} b_{(3)}}\left(\phi_{1}+\phi_{2}\right)-(2 k-5) \varphi & \rightarrow \varphi .
\end{aligned}
$$

Under these changes, the action (4.22) becomes

$$
\begin{aligned}
S= & \frac{1}{2 \pi} \int d^{2} w\left[\frac{G_{i j}^{(3)}}{2} \partial \phi^{i} \bar{\partial} \phi^{j}-\partial \varphi \bar{\partial} \varphi-\partial \chi \partial \chi+\frac{1}{4} \sqrt{g} \mathcal{R}\left(b_{(3)}\left(\phi^{1}+\phi^{2}\right)-b_{(2)} \varphi\right)\right] \\
& +\lambda \int d^{2} w \sum_{i=1}^{4} V_{i}
\end{aligned}
$$

with interaction terms

$$
\begin{array}{lll}
V_{1}=e^{\left((k-3) \phi_{1}+(k-2) \phi_{2}-\frac{2}{b_{(3)}(2)} \varphi\right) / b_{(3)}}, & V_{2}=e^{\left((k-2) \phi_{1}+(k-3) \phi_{2}-\frac{2}{\left.b_{(3)} b^{b}\right)} \varphi\right) / b_{(3)}}, \\
V_{3}=e^{\phi^{1} / b_{(3)}-\varphi / b_{(2)}+\sqrt{\frac{k}{3}} \chi}, & V_{4}=e^{\phi^{2} / b_{(3)}-\varphi / b_{(2)}-\sqrt{\frac{k}{3}} \chi} .
\end{array}
$$

They are still different from the screening charges in (2.17). For the original FZZduality, reflection relations are applied to interaction terms in order to obtain the sineLiouville theory with (1.2). We would like to act reflections also in the current case. Let us focus on a combination of interaction terms

$$
V_{3} V_{4} \sim e^{2 \phi / b_{(3)}-2 \varphi / b_{(2)}} \equiv e^{-2 i \phi^{\prime}} .
$$

Here we set

$$
\begin{aligned}
\phi & =\frac{1}{2}\left(\phi_{1}+\phi_{2}\right), & \phi_{\perp} & =\frac{1}{2 \sqrt{3}}\left(\phi_{1}-\phi_{2}\right), \\
\phi^{\prime} & =\frac{i}{b_{(3)}} \phi-\frac{i}{b_{(2)}} \varphi, & \varphi^{\prime} & =\frac{i}{b_{(2)}} \phi-\frac{i}{b_{(3)}} \varphi .
\end{aligned}
$$

The background charges for $\phi^{\prime}$ and $\varphi^{\prime}$ are

$$
Q_{\phi^{\prime}}=i, \quad Q_{\varphi^{\prime}}=2 i \frac{b_{(3)}}{b_{(2)}}-i \frac{b_{(2)}}{b_{(3)}} .
$$

The vertex operator of the form $V_{\alpha}=e^{2 \alpha \phi^{\prime}}$ satisfies a reflection relation due to the interaction term (4.27) as $V_{\alpha}=R(\alpha) V_{i-\alpha}$. Using the reflection relation, the vertex operator $\mathcal{V}_{\nu}=\tilde{V}_{\nu} V_{l_{\nu}-b_{(2)}^{-2} ; m_{\nu}, \bar{m}_{\nu}}$ in (4.21) can be replaced by ${ }^{12}$

$$
\mathcal{V}_{\nu}\left(z_{\nu}\right)=e^{b_{(3)}\left(j_{2}^{\nu} \phi_{1}+j_{1}^{\nu} \phi_{2}\right)} e^{2 b_{(2)} l^{\nu} \varphi+2 \sqrt{\frac{3}{k}}\left(m^{\nu} \chi_{L}+\bar{m}^{\nu} \chi_{R}\right)} .
$$

\footnotetext{
${ }^{12}$ If we use the reflection relation, then we need to insert the factor $R(\alpha)$. In this paper, we neglect it since it can be removed by changing the overall factor.
} 
In a similar way, the interactions $V_{1}, V_{2}$ in (4.26) are replaced by $V_{1}=e^{-\phi_{1} / b_{(3)}+2 \varphi / b_{(2)}}, V_{2}=$ $e^{-\phi_{2} / b_{(3)}+2 \varphi / b_{(2)}}$ with the reflection relation. Further acting the reflections by $V_{3}$ and $V_{4}$, respectively, the interaction terms become

$$
\begin{aligned}
& \tilde{V}_{1}=V_{3}=e^{\phi^{1} / b_{(3)}-\varphi / b_{(2)}+\sqrt{\frac{k}{3}} \chi}, \\
& \tilde{V}_{2}=V_{2}=e^{\left(\phi^{1}-\phi^{2}\right) / b_{(3)}+\varphi / b_{(2)}-\sqrt{\frac{k}{3}} \chi} \text {, } \\
& \tilde{V}_{3}=V_{1}=e^{\left(\phi^{2}-\phi^{1}\right) / b_{(3)}+\varphi / b_{(2)}+\sqrt{\frac{k}{3}} \chi} \text {, } \\
& \tilde{V}_{4}=V_{4}=e^{\phi^{2} / b_{(3)}-\varphi / b_{(2)}-\sqrt{\frac{k}{3}} \chi} \text {. }
\end{aligned}
$$

They are the same as the screening operators in (2.17) with $N=2$ as desired. ${ }^{13}$

We can explicitly check that the dual theory has the same symmetry algebra as that of the coset model (3.1). The symmetry generators of the theory are written by the four fields $\left(\phi_{1}, \phi_{2}, \varphi, \chi\right)$ and their OPEs with $\tilde{V}_{i}$ should be given by total derivatives. There are no spin-one currents satisfying these conditions. We find a spin-two current, which is the energy-momentum tensor,

$$
T=-\frac{1}{2} G_{i j}^{(3)} \partial \phi^{i} \partial \phi^{j}+b_{(3)} \partial^{2}\left(\phi^{1}+\phi^{2}\right)+\partial \varphi \partial \varphi-b_{(2)} \partial^{2} \varphi+\partial \chi \partial \chi .
$$

Its central charge reproduces (1.4) with $N=2$. In a similar way, we find a spin-three current and a spin-four current. The ratio of OPE coefficients in (2.6) reproduces (2.8) with $N=2$.

\section{A higher rank generalization}

In the previous sections, we have examined the coset model (1.3) with $N=2$ and its duality. In this section, we extend the analysis for the coset (1.3) with $N=3$,

$$
\frac{\mathfrak{s l}(4)_{k}}{\mathfrak{s l}(3)_{k} \times \mathfrak{u}(1)}
$$

In the $N=2$ case, the reduction procedure itself was rather straightforward. Namely, we just integrated the ghost sector and performed the shifts of other fields. It turns out that the situation is more complicate for a higher rank case. A main point of [33] was to utilize a map between different free field realizations for same non-regular $\mathrm{W}$-algebra, and the same technique is needed for the current case as we explain in this section.

\subsection{First order formulation of coset model}

As in the previous case, we start by finding out a first order formulation of the coset model. For this, we first express the $\mathfrak{s l}(4)$ current algebra in terms of free fields and then consider

\footnotetext{
${ }^{13}$ It might be convenient to redefine $\tilde{X}=\tilde{X}_{L}-\tilde{X}_{R}=-i\left(\chi_{L}+\chi_{R}\right)$ in order to compare with the interaction terms of sine-Liouville theory in (1.2). Here we choose to use $\chi_{L}, \chi_{R}$ to make the $\mathfrak{s l}(N+1 \mid N)$ structure clearer.
} 
the subsector orthogonal to the denominator algebra of the coset (5.1). We use the action of $\mathfrak{s l}(4)$ WZNW model in the first order formulation,

$$
\begin{aligned}
S= & \frac{1}{2 \pi} \int d^{2} w\left[\frac{G_{i j}^{(4)}}{2} \partial \phi^{i} \bar{\partial} \phi^{j}+\frac{b_{(4)}}{4} \sqrt{g} \mathcal{R} \sum_{i=1}^{3} \phi^{i}+\sum_{\alpha=1}^{6}\left(\beta_{\alpha} \bar{\partial} \gamma_{\alpha}+\bar{\beta}_{\alpha} \partial \bar{\gamma}_{\alpha}\right)\right] \\
& -\frac{1}{2 \pi k} \int d^{2} w\left[e^{b_{(4)} \phi_{1}}\left|\beta_{1}\right|^{2}+e^{b_{(4)} \phi_{2}}\left|\beta_{2}-\gamma_{1} \beta_{4}\right|^{2}+e^{b_{(4)} \phi_{3}}\left|\beta_{3}-\gamma_{2} \beta_{5}-\gamma_{4} \beta_{6}\right|^{2}\right],
\end{aligned}
$$

see, e.g., (5.2) of [33]. Here we set $b_{(4)}=1 / \sqrt{k-4}$ as in (2.18) with $a=4$ and use

$$
G_{i j}^{(4)}=\left(\begin{array}{ccc}
2 & -1 & 0 \\
-1 & 2 & -1 \\
0 & -1 & 2
\end{array}\right), \quad G^{(4) i j}=\left(\begin{array}{ccc}
3 / 4 & 1 / 2 & 1 / 4 \\
1 / 2 & 1 & 1 / 2 \\
1 / 4 & 1 / 2 & 3 / 4
\end{array}\right)
$$

Among the $\mathfrak{s l}(4)$ currents, the Cartan directions are generated by

$$
\begin{aligned}
& h^{1}=b_{(4)}^{-1} \partial \phi_{1}+2 \gamma_{1} \beta_{1}-\gamma_{2} \beta_{2}+\gamma_{4} \beta_{4}-\gamma_{5} \beta_{5}+\gamma_{6} \beta_{6}, \\
& h^{2}=b_{(4)}^{-1} \partial \phi_{2}-\gamma_{1} \beta_{1}+2 \gamma_{2} \beta_{2}-\gamma_{3} \beta_{3}+\gamma_{4} \beta_{4}+\gamma_{5} \beta_{5}, \\
& h^{3}=b_{(4)}^{-1} \partial \phi_{3}-\gamma_{2} \beta_{2}+2 \gamma_{3} \beta_{3}-\gamma_{4} \beta_{4}+\gamma_{5} \beta_{5}+\gamma_{6} \beta_{6} .
\end{aligned}
$$

In order to construct the coset theory, we identify the generators of $\mathfrak{s l}(3)$ subalgebra with

$$
\hat{e}^{1}=\beta_{4}-\gamma_{3} \beta_{6}, \quad \hat{e}^{2}=\beta_{3}, \quad \hat{e}^{3}=\beta_{6}, \quad \hat{h}^{1}=h^{1}+h^{2}, \quad \hat{h}^{2}=h^{3} .
$$

The other generators $\hat{f}^{1}, \hat{f}^{2}, \hat{f}^{3}$ can be constructed from the above generators. The generator of $\mathfrak{u}(1)$ subalgebra is

$$
\hat{h}^{\prime}=h^{1}-2 h^{2}-h^{3} .
$$

We introduce new fields by

$$
\begin{aligned}
b_{(3)}^{-1} \partial \hat{\varphi}_{1} & =b_{(4)}^{-1}\left(\partial \phi_{1}+\partial \phi_{2}\right)+\gamma_{1} \beta_{1}+\gamma_{2} \beta_{2}, \\
b_{(3)}^{-1} \partial \hat{\varphi}_{2} & =b_{(4)}^{-1} \partial \phi_{3}-\gamma_{2} \beta_{2}+\gamma_{5} \beta_{5}, \\
2 \sqrt{6 k} \partial \hat{\chi} & =b_{(4)}^{-1}\left(\partial \phi_{1}-2 \partial \phi_{2}-\partial \phi_{3}\right)+4\left(\gamma_{1} \beta_{1}-\gamma_{2} \beta_{2}-\gamma_{5} \beta_{5}\right) .
\end{aligned}
$$

We then consider the subspace orthogonal to $\hat{\varphi}_{1}, \hat{\varphi}_{2}, \hat{\chi}$ and neglect the ghost systems $\left(\gamma_{\alpha}, \beta_{\alpha}\right)$ with $\alpha=3,4,6$.

Introducing new fields $\varphi_{1}, \varphi_{2}, \chi$ to cancel the contributions from $\hat{\varphi}_{1}, \hat{\varphi}_{2}, \hat{\chi}$, the action of the coset theory is given by

$$
\begin{aligned}
S= & \frac{1}{2 \pi} \int d^{2} w\left[\frac{G_{i j}^{(4)}}{2} \partial \phi^{i} \bar{\partial} \phi^{j}-\frac{G_{i j}^{(3)}}{2} \partial \varphi^{i} \bar{\partial} \varphi^{j}-\partial \chi \bar{\partial} \chi\right] \\
& +\frac{1}{2 \pi} \int d^{2} w\left[\frac{1}{4} \sqrt{g} \mathcal{R}\left(b_{(4)} \sum_{i=1}^{3} \phi^{i}-b_{(3)} \sum_{i=1}^{2} \varphi^{i}\right)+\sum_{\alpha=1,2,5}\left(\beta_{\alpha} \bar{\partial} \gamma_{\alpha}+\bar{\beta}_{\alpha} \partial \bar{\gamma}_{\alpha}\right)\right] \\
& -\frac{1}{2 \pi k} \int d^{2} w\left[e^{b_{(4)} \phi_{1}}\left|\beta_{1}\right|^{2}+e^{b_{(4)} \phi_{2}}\left|\beta_{2}\right|^{2}+e^{b_{(4)} \phi_{3}}\left|\gamma_{2} \beta_{5}\right|^{2}\right] .
\end{aligned}
$$


Here one may notice that an interaction term depends on $\gamma_{2}, \bar{\gamma}_{2}$, which makes difficult to integrate $\beta_{2}, \bar{\beta}_{2}$ out. The vertex operators are of the form

$$
\Psi(z)=\left[\prod_{\alpha=1,2,5} \gamma_{\alpha}^{r_{\alpha}} \bar{\gamma}_{\alpha}^{\bar{r}_{\alpha}}\right] e^{b_{(4)}\left(j_{1} \phi_{1}+j_{2} \phi_{2}+j_{3} \phi_{3}\right)} e^{b_{(3)}\left(l_{1} \varphi_{1}+l_{2} \varphi_{2}\right)+2 \sqrt{\frac{6}{k}}\left(m \chi_{L}+\bar{m} \chi_{R}\right)}
$$

with

$$
r_{1}=-j_{1}-l_{1}-m, \quad r_{2}=-j_{2}+j_{3}-l_{1}+l_{2}+m, \quad r_{5}=-j_{3}-l_{2}+m
$$

and similarly for $\bar{r}_{1}, \bar{r}_{2}, \bar{r}_{5}$.

The spectral flow operator $v^{\left\{S_{i}\right\}}(\xi)$ can be constructed as explained in subsection 3.3. In the current case, there are three parameters $S_{i}$ with $i=1,2,3$. Here we choose the parameters such that $\beta_{\alpha}$ with $\alpha=1,2,5$ have zeros of order $S_{i}$ with $i=1,2,3$, respectively, at $w=\xi$ after the insertion of the operator. Along with them, there are similar requirements for $\beta_{\alpha}(\alpha=3,4,6)$. It induces the insertion of $e^{\sum_{i=1}^{3} S_{i} \phi^{i} / b_{(4)}}(\xi)$ at the same time.

\subsection{Reduction from coset theory}

We compute the correlation functions of coset theory with vertex operators of the form (5.9). It is convenient to express them as

$$
\begin{aligned}
& \Psi_{\nu}\left(z_{\nu}\right)=\int \frac{d^{2} \mu_{2}^{\nu}}{\left|\mu_{2}^{\nu}\right|^{2}}\left(\mu_{2}^{\nu}\right)^{-r_{2}^{\nu}}\left(\bar{\mu}_{2}^{\nu}\right)^{-\bar{r}_{2}^{\nu}} \tilde{\Psi}_{\nu}\left(z_{\nu}\right), \\
& \tilde{\Psi}_{\nu}\left(z_{\nu}\right)=\Phi_{\nu}\left(z_{\nu}\right) V_{l_{1}^{\prime}, l_{2}^{\nu} ; m^{\nu}, \bar{m}^{\nu}}^{\varphi_{1}, \varphi_{2}, \chi}\left(z_{\nu}\right)
\end{aligned}
$$

with $r_{2}^{\nu}$ as in (5.10). Here we have defined $\Phi_{\nu}\left(z_{\nu}\right)$ as

$$
\begin{aligned}
& \Phi_{\nu}\left(z_{\nu}\right)=\int \frac{d^{2} \mu_{1}^{\nu} d^{2} \mu_{5}^{\nu}}{\left|\mu_{1}^{\nu}\right|^{2}\left|\mu_{5}^{\nu}\right|^{2}}\left(\mu_{1}^{\nu}\right)^{l_{1}^{\nu}+m^{\nu}}\left(\bar{\mu}_{1}^{\nu}\right)^{\nu}+\bar{m}^{\nu}\left(\mu_{5}^{\nu}\right)^{l_{2}^{\nu}-m^{\nu}}\left(\bar{\mu}_{5}^{\nu}\right)^{l_{2}^{\nu}-\bar{m}^{\nu}} V_{\nu}\left(z_{\nu}\right), \\
& V_{\nu}\left(z_{\nu}\right)=\left|\mu_{1}^{\nu}\right|^{2 j_{1}^{\nu}}\left|\mu_{5}^{\nu}\right|^{2 j_{3}^{\nu}} e^{\sum_{\alpha=1,2,5}\left(\mu_{\alpha}^{\nu} \gamma_{\alpha}-\bar{\mu}_{\alpha}^{\nu} \bar{\gamma}_{\alpha}\right)} e^{b_{(4)}\left(j_{1}^{\nu} \phi_{1}+j_{2}^{\nu} \phi_{2}+j_{3}^{\nu} \phi_{3}\right)}\left(z_{\nu}\right),
\end{aligned}
$$

and $V_{l_{1}^{\prime}, l_{2} ; m^{\nu}, \bar{m}^{\nu}}^{\varphi_{1}, \varphi_{2}, \chi}\left(z_{\nu}\right)$ as

$$
V_{l_{1}^{\nu}, l_{2}^{\nu} ; m^{\nu}, \bar{m}^{\nu}}^{\varphi_{1}, \varphi_{2}, \chi}\left(z_{\nu}\right)=e^{b_{(3)}\left(l_{1}^{\nu} \varphi_{1}+l_{2}^{\nu} \varphi_{2}\right)+2 \sqrt{\frac{6}{k}}\left(m^{\nu} \chi_{L}+\bar{m}^{\nu} \chi_{R}\right)} .
$$

We first focus on the part of $\mathfrak{s l}(4)$ WZNW model. The vertex operators do not have any dependence on $\gamma_{3}, \gamma_{4}, \gamma_{6}$, which means that we can set $\beta_{\alpha}=0$ with $\alpha=3,4,6$. We would like to perform the reduction procedure by integrating out $\left(\gamma_{\alpha}, \beta_{\alpha}\right)$ with $\alpha=1,2,5$. However, as noticed above, it is difficult to do so for $\left(\gamma_{2}, \beta_{2}\right)$ because of the existence of $\gamma_{2}$ in an interaction term of (5.8). In this subsection, we only consider the correlation functions of $\tilde{\Psi}_{\nu}\left(z_{\nu}\right)$ in (5.12). We will later perform the integration over $\left(\gamma_{2}, \beta_{2}\right)$.

We thus start from the correlation functions of $\mathfrak{s l}(4)$ WZNW model in the form of

$$
\begin{aligned}
& \left\langle\prod_{\nu=1}^{N} V_{\nu}\left(z_{\nu}\right) v^{\left\{S_{j}\right\}}(\xi)\right\rangle \\
& \quad=\int_{S_{j}, \xi}\left[\prod_{i=1}^{3} \mathcal{D} \phi_{i}\right]\left[\prod_{\alpha=1,2,5} \mathcal{D}^{2} \beta_{\alpha} \mathcal{D}^{2} \gamma_{\alpha}\right] e^{-S} \prod_{\nu=1}^{N} V_{\nu}\left(z_{\nu}\right) e^{\sum_{i=1}^{3} S_{i} \phi^{i} / b_{(4)}}(\xi),
\end{aligned}
$$


where $S$ denotes the action of $\mathfrak{s l}(4)$ WZNW model in (5.2) with $\beta_{\alpha}=0(\alpha=3,4,6)$. We integrate out $\gamma_{i}, \beta_{i}(i=1,5)$ and keep $\gamma_{2}, \beta_{2}$ as it is for a while. We also set $S_{2}=0$. Taking care of the insertion of $v^{\left\{S_{j}\right\}}(\xi)$, we find that $\beta_{1}, \beta_{5}$ are replaced by functions as

$$
\begin{aligned}
& \beta_{1}(w)=-\sum_{\nu=1}^{N} \frac{\mu_{1}^{\nu}}{w-z_{\nu}}=-u_{1} \frac{(w-\xi)^{S_{1}} \prod_{p=1}^{N-2-S_{1}}\left(w-y_{p}^{1}\right)}{\prod_{\nu=1}^{N}\left(w-z_{\nu}\right)} \equiv-u_{1} \mathcal{B}_{1}\left(w ; z_{\nu}, y_{p}^{1}\right), \\
& \beta_{5}(w)=-\sum_{\nu=1}^{N} \frac{\mu_{5}^{\nu}}{w-z_{\nu}}=-u_{3} \frac{(w-\xi)^{S_{3}} \prod_{p=1}^{N-2-S_{3}}\left(w-y_{p}^{3}\right)}{\prod_{\nu=1}^{N}\left(w-z_{\nu}\right)} \equiv-u_{3} \mathcal{B}_{3}\left(w ; z_{\nu}, y_{p}^{3}\right) .
\end{aligned}
$$

There are also constraints as

$$
\begin{aligned}
& \sum_{\nu=1}^{N} \frac{\mu_{1}^{\nu}}{\left(\xi-z_{\nu}\right)^{n}}=0 \quad\left(n=0,1, \ldots, S_{1}\right), \\
& \sum_{\nu=1}^{N} \frac{\mu_{5}^{\nu}}{\left(\xi-z_{\nu}\right)^{n}}=0 \quad\left(n=0,1, \ldots, S_{3}\right) .
\end{aligned}
$$

As before, we remove functions in interaction terms by shifting fields as

$$
\phi_{1}+\frac{1}{b_{(4)}} \ln \left|u_{1} \mathcal{B}_{1}\right| \rightarrow \phi_{1}, \quad \phi_{3}+\frac{1}{b_{(4)}} \ln \left|u_{3} \mathcal{B}_{3}\right| \rightarrow \phi_{3} .
$$

There are several contributions from the kinetic terms in particular and the correlation function (5.16) becomes

$$
\begin{aligned}
\left\langle\prod_{\nu=1}^{N} V_{\nu}\left(z_{\nu}\right) v^{\left\{S_{j}\right\}}(\xi)\right\rangle= & \left|\Theta_{N}\right|^{2} \prod_{n=0}^{S_{1}} \delta^{(2)}\left(\sum_{\nu=1}^{N} \frac{\mu_{1}^{\nu}}{\left(\xi-z_{\nu}\right)^{n}}\right) \prod_{n=0}^{S_{3}} \delta^{(2)}\left(\sum_{\nu=1}^{N} \frac{\mu_{5}^{\nu}}{\left(\xi-z_{\nu}\right)^{n}}\right) \\
& \times\left\langle\prod_{\nu=1}^{N} \tilde{V}_{\nu}\left(z_{\nu}\right) \prod_{p=1}^{N-2-S_{1}} \tilde{V}_{b}\left(y_{p}^{1}\right) \prod_{p=1}^{N-2-S_{3}} \tilde{V}_{b}\left(y_{p}^{3}\right)\right\rangle .
\end{aligned}
$$

The right hand side is evaluated with the action

$$
\begin{aligned}
S= & \frac{1}{2 \pi} \int d^{2} w\left[\frac{G_{i j}^{(4)}}{2} \partial \phi^{i} \bar{\partial} \phi^{j}+\frac{1}{4} \sqrt{g} \mathcal{R}\left(\left(b_{(4)}+b_{(4)}^{-1}\right)\left(\phi^{1}+\phi^{3}\right)+b_{(4)} \phi^{2}\right)\right] \\
& +\frac{1}{2 \pi} \int d^{2} w\left[\beta_{2} \bar{\partial} \gamma_{2}+\bar{\beta}_{2} \partial \bar{\gamma}_{2}+\frac{1}{k}\left(e^{b_{(4)} \phi_{1}}-e^{b_{(4)} \phi_{2}}\left|\beta_{2}\right|^{2}+e^{b_{(4)} \phi_{3}}\left|\gamma_{2}\right|^{2}\right)\right] .
\end{aligned}
$$

The vertex operators are modified as

$$
\tilde{V}_{\nu}\left(z_{\nu}\right)=e^{\mu_{2}^{\nu} \gamma_{2}-\bar{\mu}_{2}^{\nu} \bar{\gamma}_{2}} e^{b_{(4)}\left(j_{1}^{\nu} \phi_{1}+j_{2}^{\nu} \phi_{2}+j_{3}^{\nu} \phi_{3}\right)+\left(\phi^{1}+\phi^{3}\right) / b_{(4)}}
$$

and the inserted vertex operators are

$$
\tilde{V}_{b}\left(y_{p}^{1}\right)=e^{-\phi^{1} / b_{(4)}}, \quad \tilde{V}_{b}\left(y_{p}^{3}\right)=e^{-\phi^{3} / b_{(4)}} .
$$

The pre-factor is computed as

$$
\begin{aligned}
& \Theta_{N}=u_{1}{ }^{3-\frac{3 S_{1}+S_{3}}{4 b_{(4)}^{2}}} u_{3} 3-\frac{S_{1}+3 S_{3}}{4 b_{(4)}^{2}} \\
& \times\left[\prod_{\nu<\nu^{\prime}} z_{\nu \nu^{\prime}}\right]^{\frac{2}{b_{(4)}^{2}}}\left[\prod_{p, \nu}\left(z_{\nu}-y_{p}^{1}\right)\left(z_{\nu}-y_{p}^{3}\right)\right]^{-\frac{1}{b_{(4)}^{2}}}\left[\prod_{p, p^{\prime}}\left(y_{p}^{1}-y_{p^{\prime}}^{3}\right)\right]^{\frac{1}{4 b_{(4)}^{2}}}\left[\prod_{p<p^{\prime}} y_{p p^{\prime}}^{1} y_{p p^{\prime}}^{3}\right]^{\frac{3}{4 b_{(4)}^{2}}} .
\end{aligned}
$$


We then apply the analysis to our coset theory with the action (5.8) and the vertex operators of the form (5.12). For the coset correlation function, we may insert an identity operator

$$
\mathbb{1}=v^{\left\{S_{j}\right\}}(\xi) e^{-\left(S_{1} \varphi^{1}+S_{3} \varphi^{2}\right) / b_{(3)}}(\xi) e^{-\left(S_{1}-S_{3}\right) \sqrt{\frac{k}{6}}\left(\chi_{L}+\chi_{R}\right)}(\xi) .
$$

Applying the reduction produce for the part of $\mathfrak{s l}(4)$ WZNW model, the parameters $\mu_{1}^{\nu}, \mu_{5}^{\nu}$ are mapped to $y_{p}^{1}, y_{p}^{3}$. We further shift $\varphi_{1}, \varphi_{2}, \chi$ as

$$
\begin{aligned}
\varphi_{1}+\frac{1}{b_{(3)}} \ln \left|u_{1} \mathcal{B}_{1}\right|^{2} & \rightarrow \varphi_{1}, & \varphi_{2}+\frac{1}{b_{(3)}} \ln \left|u_{3} \mathcal{B}_{3}\right|^{2} & \rightarrow \varphi_{2}, \\
\chi_{L}+\frac{1}{2} \sqrt{\frac{k}{6}}\left[\ln \left(u_{1} \mathcal{B}_{1}\right)-\ln \left(u_{3} \mathcal{B}_{3}\right)\right] & \rightarrow \chi_{L}, & \chi_{R}+\frac{1}{2} \sqrt{\frac{k}{6}}\left[\ln \left(\bar{u}_{1} \overline{\mathcal{B}}_{1}\right)-\ln \left(\bar{u}_{3} \overline{\mathcal{B}}_{3}\right)\right] & \rightarrow \chi_{R}
\end{aligned}
$$

in order to remove $u_{1} \mathcal{B}_{1}^{\nu}, u_{1} \mathcal{B}_{5}^{\nu}$ appearing in (5.12) with (5.13). The correlation function is now expressed as

$$
\left\langle\prod_{\nu=1}^{N} \tilde{\Psi}_{\nu}\left(z_{\nu}\right)\right\rangle=\left\langle\prod_{\nu=1}^{N} \tilde{V}_{\nu}\left(z_{\nu}\right) V_{l_{1}^{\nu}-b_{(3)}^{-2}, l_{2}^{\nu}-b_{(3)}^{-2} ; m^{\nu}, \bar{m}^{\nu}}^{\left.\varphi_{1}, z_{\nu}\right)}\right\rangle .
$$

The right hand side is evaluated with the action

$$
\begin{aligned}
S= & \frac{1}{2 \pi} \int d^{2} w\left[\frac{G_{i j}^{(4)}}{2} \partial \phi^{i} \bar{\partial} \phi^{j}-\frac{G_{i j}^{(3)}}{2} \partial \varphi^{i} \bar{\partial} \varphi^{j}-\partial \chi \bar{\partial} \chi\right] \\
& +\frac{1}{2 \pi} \int d^{2} w\left[\frac{1}{4} \sqrt{g} \mathcal{R}\left(\sum_{i=1}^{3} Q_{\phi, i} \phi^{i}-\sum_{i=1}^{2} Q_{\varphi, i} \varphi^{i}\right)+\beta_{2} \bar{\partial} \gamma_{2}+\bar{\beta}_{2} \partial \bar{\gamma}_{2}\right] \\
& +\frac{1}{2 \pi} \int d^{2} w\left[\frac{1}{k}\left(e^{b_{(4)} \phi_{1}}-e^{b_{(4)} \phi_{2}}\left|\beta_{2}\right|^{2}+e^{b_{(4)} \phi_{3}}\left|\gamma_{2}\right|^{2}\right)+2 \pi \lambda\left(V_{1}+V_{2}\right)\right],
\end{aligned}
$$

where

$$
Q_{\phi, 1}=Q_{\phi, 3}=b_{(4)}+\frac{1}{b_{(4)}}, \quad Q_{\phi, 2}=b_{(4)}, \quad Q_{\varphi, 1}=Q_{\varphi, 2}=b_{(3)}+\frac{1}{b_{(3)}}
$$

and

$$
V_{1}=e^{-\phi^{1} / b_{(4)}+\varphi^{1} / b_{(3)}+\sqrt{\frac{k}{6}}\left(\chi_{L}+\chi_{R}\right)}, \quad V_{2}=e^{-\phi^{3} / b_{(4)}+\varphi^{2} / b_{(3)}-\sqrt{\frac{k}{6}}\left(\chi_{L}+\chi_{R}\right)} .
$$

In order to obtain (5.27), we have regarded the vertex operators inserted at $w=y_{p}^{1}, y_{p}^{3}$ as interaction terms as before.

\subsection{Interpretation as extended BP-theory}

As mentioned above, we have to deal with $\gamma_{2}$ in an interaction term of the action (5.28) in order to go furthermore. We would like to apply the technique developed in [33]. For this, it is convenient to make a change of variables as

$$
x=\phi_{1}+\frac{2}{3} \phi_{2}+\frac{1}{3} \phi_{3}, \quad x_{1}=\phi_{2}, \quad x_{2}=\phi_{3} .
$$


The action is now

$$
\begin{aligned}
S= & \frac{1}{2 \pi} \int d^{2} w\left[\frac{3}{8} \partial x \bar{\partial} x+\frac{G_{i j}^{(3)}}{2} \partial x^{i} \bar{\partial} x^{j}-\frac{G_{i j}^{(3)}}{2} \partial \varphi^{i} \bar{\partial} \varphi^{j}-\partial \chi \bar{\partial} \chi\right] \\
& +\frac{1}{2 \pi} \int d^{2} w\left[\frac{1}{4} \sqrt{g} \mathcal{R}\left(Q_{x} x+\sum_{i=1}^{2} Q_{x, i} x^{i}-\sum_{i=1}^{2} Q_{\varphi, i} \varphi^{i}\right)+\beta_{2} \bar{\partial} \gamma_{2}+\bar{\beta}_{2} \partial \bar{\gamma}_{2}\right] \\
& +\frac{1}{2 \pi} \int d^{2} w\left[\frac{1}{k}\left(-e^{b_{(4)} x_{1}}\left|\beta_{2}\right|^{2}+e^{b_{(4)} x_{2}}\left|\gamma_{2}\right|^{2}\right)+2 \pi \lambda \sum_{i=0}^{2} V_{i}\right]
\end{aligned}
$$

The background charges are

$$
\begin{aligned}
Q_{x} & =\frac{3}{2} b_{(4)}+\frac{1}{b_{(4)}}, \quad Q_{x, 1}=b_{(4)}, \quad Q_{x, 2}=b_{(4)}+\frac{1}{b_{(4)}}, \\
Q_{\varphi, 1} & =Q_{\varphi, 2}=b_{(3)}+\frac{1}{b_{(3)}}
\end{aligned}
$$

and the interaction terms are

$$
\begin{aligned}
V_{0} & =e^{\left(x-\frac{2}{3} x_{1}-\frac{1}{3} x_{2}\right) / b_{(4)}}, \\
V_{1} & =e^{-\frac{3}{4} x / b_{(4)}+\varphi^{1} / b_{(3)}+\sqrt{\frac{k}{6}}\left(\chi_{L}+\chi_{R}\right)} \\
V_{2} & =e^{-\left(\frac{1}{4} x+\frac{1}{3} x_{1}+\frac{2}{3} x_{2}\right) / b_{(4)}+\varphi^{2} / b_{(3)}-\sqrt{\frac{k}{6}}\left(\chi_{L}+\chi_{R}\right)} .
\end{aligned}
$$

We would like to evaluate correlation functions of the vertex operators of the form (5.11). From the relation of correlation functions (5.27) and the change of variables (5.31), the vertex operators are now given by

$$
\Psi_{\nu}^{\prime}\left(z_{\nu}\right)=\int \frac{d^{2} \mu_{2}^{\nu}}{\left|\mu_{2}^{\nu}\right|^{2}}\left(\mu_{2}^{\nu}\right)^{-r_{2}^{\nu}}\left(\bar{\mu}_{2}^{\nu}\right)^{-\bar{r}_{2}^{\nu}} \tilde{V}_{\nu}\left(z_{\nu}\right) V_{l_{1}^{\nu}-b_{(3)}^{-2}, l_{2}^{\nu}-b_{(3)}^{-2} ; m^{\nu}, \bar{m}^{\nu}}^{\left.\varphi_{1}, z_{\nu}\right)}
$$

with

$$
\tilde{V}_{\nu}\left(z_{\nu}\right)=e^{\mu_{2}^{\nu} \gamma_{2}-\bar{\mu}_{2}^{\nu} \bar{\gamma}_{2}} e^{\left(b_{(4)} j_{1}^{\nu}+\frac{1}{b_{(4)}}\right) x+\left(b_{(4)}\left(j_{2}^{\nu}-\frac{2}{3} j_{1}^{\nu}\right)+\frac{1}{3 b_{(4)}}\right) x_{1}+\left(b_{(4)}\left(j_{3}^{\nu}-\frac{1}{3} j_{1}^{\nu}\right)+\frac{2}{3 b_{(4)}}\right) x_{2}} .
$$

As seen in appendix B, the first two interaction terms in the action (5.32) correspond to screening operators of BP-algebra in a free field realization. Following [33], we shall remove the $\gamma_{2}$-dependence of interaction term by exchanging the free field realizations of BP-algebra.

For $V_{0}$, we have used the self-duality with respect to a Liouville field, which may require explanation. Let us go back to the action (5.28) and perform the change of variables as

$$
\phi_{1}^{\prime}=\frac{1}{2} \phi_{1}, \quad \phi_{2}^{\prime}=\frac{1}{2} \phi_{1}+\phi_{2}, \quad \phi_{3}^{\prime}=\phi_{3} .
$$

Then the new field $\phi_{1}^{\prime}$ does not have any non-trivial OPEs with the other new fields and its background charge is $Q=b_{(4)}+b_{(4)}^{-1}$. The term $e^{2 b_{(4)} \phi_{1}^{\prime}}$ can be regarded as the interaction term of Liouville field theory with $\phi_{1}^{\prime}$. Therefore, applying the self-duality of the Liouville 
field theory, the interaction term can be replaced by $e^{2 \phi_{1}^{\prime} / b_{(4)}}$. Going back to the original fields $\phi_{i}$ and applying the change of variables in (5.31), we arrive at the expression with $V_{0}$ in (5.34).

We shall treat the theory with the action (5.32) as an extension of the theory with BP-algebra symmetry. The action of the BP-theory is

$$
\begin{aligned}
S= & \frac{1}{2 \pi} \int d^{2} w\left[\frac{G_{i j}^{(3)}}{2} \partial x^{i} \bar{\partial} x^{j}+\frac{1}{4} \sqrt{g} \mathcal{R} \sum_{i=1}^{2} Q_{x, i} x^{i}\right] \\
& -\lambda \int d^{2} w\left[e^{b_{(4)} x_{1}}\left|\beta_{2}\right|^{2}-e^{b_{(4)} x_{2}}\left|\gamma_{2}\right|^{2}\right]
\end{aligned}
$$

where $Q_{x, 1}, Q_{x, 2}$ are given in (5.33). The interaction terms correspond to screening charges (B.4) in a free field realization of BP-algebra. In particular, the spin-one current for the BP-algebra is written in terms of free fields as

$$
H=\frac{1}{b_{(4)}}\left(\partial x^{2}-\partial x^{1}\right)-\gamma_{2} \beta_{2},
$$

see (B.5). We would like to move to the theory corresponding to the other free field realization of BP-algebra. From the expression of screening operators in (B.6), the action should be given by

$$
\begin{aligned}
S= & \frac{1}{2 \pi} \int d^{2} w\left[\frac{G_{i j}^{(3)}}{2} \partial x^{i} \bar{\partial} x^{j}+\frac{1}{4} \sqrt{g} \mathcal{R} \sum_{i=1}^{2} Q_{x, i} x^{i}\right] \\
& -\lambda \int d^{2} w\left[e^{b_{(4)} x_{1}}\left|\beta_{2}\right|^{2}-e^{b_{(4)} x_{2}}\right] .
\end{aligned}
$$

In this case, the spin-one current for the BP-algebra is expressed as

$$
H=\frac{1}{b_{(4)}} \partial x^{1}+\gamma_{2} \beta_{2},
$$

see (B.7).

In order to move from one description with (5.38) to the other one with (5.40), we should change the vertex operators such as to behave in the same way under the action of BP-algebra generators. In the current analysis, we neglect overall factors, so it is enough to have the same charge with respect to the spin-one current $H$. For more details, see appendix $\mathrm{B}$ and [33]. For $V_{1}$ and $V_{2}$, we can use the same vertex operators since they have the same $H$-charge for the both descriptions. For $V_{0}$ and $\Psi_{\nu}^{\prime}$, we should replace them by

$$
\begin{aligned}
V_{0}^{\prime} & =\int \frac{d^{2} \mu_{2}}{\left|\mu_{2}\right|^{2}}\left|\mu_{2}\right|^{-\frac{2}{3 b_{(4)}^{2}}} e^{\mu_{2} \gamma_{2}-\bar{\mu}_{2} \bar{\gamma}_{2}} e^{\left(x-\frac{2}{3} x_{1}-\frac{1}{3} x_{2}\right) / b_{(4)}}, \\
\Psi_{\nu}^{\prime \prime}\left(z_{\nu}\right) & =\int \frac{d^{2} \mu_{2}^{\nu}}{\left|\mu_{2}^{\nu}\right|^{2}}\left(\mu_{2}^{\nu}\right)^{-\tilde{r}_{2}^{\nu}}\left(\bar{\mu}_{2}^{\nu}\right)^{-\tilde{r}_{2}^{\nu}} \tilde{V}_{\nu}\left(z_{\nu}\right) V_{l_{1}^{\nu}-b_{(3)}^{-2}, l_{2}^{\nu}-b_{(3)}^{-2} ; m^{\nu}, \bar{m}^{\nu}}^{\left.\varphi_{1}, z_{\nu}\right)}
\end{aligned}
$$

with

$$
\tilde{r}_{2}^{\nu}=l_{1}^{\nu}-l_{2}^{\nu}-m^{\nu}+j_{1}^{\nu}-j_{2}^{\nu}, \quad \bar{r}_{2}^{\nu}=l_{1}^{\nu}-l_{2}^{\nu}-\bar{m}^{\nu}+j_{1}^{\nu}-j_{2}^{\nu}
$$

in order to have the same $H$-charges after the change of description. 


\subsection{Reduction from extended BP-theory}

Now we can use the action, where the interaction terms do not depend on $\gamma_{2}$. Thus, we can integrate $\left(\gamma_{2}, \beta_{2}\right)$ out and apply the reduction procedure. During the change of description, one of the interaction terms, $V_{0}$, is replaced by $V_{0}^{\prime}$ in (5.42), which has now $\gamma_{2}$-dependence. We are implicitly treating the interaction term $V_{0}^{\prime}$ in a perturbative way. Namely, we insert vertex operators at $w=z_{\nu}$ and integrate over $\mu_{2}^{\nu}$, where we have set $\nu=n+1, n+2, \ldots, n+s$. The theory may be regarded as a theory with BP-algebra symmetry but gauged by its $\mathfrak{u}(1)$ subalgebra generated by $H$. For the vertex operators at $w=z_{\nu}$, the $H$-charge is canceled by the one generated by

$$
\hat{H}=\frac{1}{4 b_{(4)}} \partial x-\frac{1}{b_{(3)}}\left(\partial \varphi^{1}-\partial \varphi^{2}\right)+\sqrt{\frac{k}{6}} \partial \chi_{L} .
$$

With the interpretation, an identity operator may be represented as

$$
\mathbb{1}=v^{S_{2}}(\xi) e^{-S_{2} x /\left(4 b_{(4)}\right)}(\xi) e^{S_{2}\left(\varphi^{1}-\varphi^{2}\right) / b_{(3)}}(\xi) e^{-S_{2} \sqrt{\frac{k}{6}}\left(\chi_{L}+\chi_{R}\right)}(\xi) .
$$

Here $v^{S_{2}}(\xi)$ induces the insertion of $e^{S_{2} x^{1} / b_{(4)}}(\xi)$ and puts a restriction on the domain of integration over $\beta_{2}$ such as to have a zero of order $S_{2}$ at $w=\xi$.

Integration over $\gamma_{2}, \beta_{2}$ leads to the replacement

$$
\beta_{2}(w)=-\sum_{\nu=1}^{N+s} \frac{\mu_{2}^{\nu}}{w-z_{\nu}}=-u_{2} \frac{(w-\xi)^{S_{2}} \prod_{p=1}^{N+s-2-S_{2}}\left(w-y_{p}^{2}\right)}{\prod_{\nu=1}^{N+s}\left(w-z_{\nu}\right)} \equiv-u_{2} \mathcal{B}\left(w ; z_{\nu}, y_{p}^{2}\right)
$$

subject to the constraints

$$
\sum_{\nu=1}^{N+s} \frac{\mu_{2}^{\nu}}{\left(\xi-z_{\nu}\right)^{n}}=0
$$

for $n=0,1, \ldots, S_{2}$. We then perform the shifts of fields as

$$
\begin{aligned}
x_{1}+\frac{1}{b_{(4)}} \ln \left|u_{2} \mathcal{B}_{2}\right|^{2} & \rightarrow x_{1}, \quad x-\frac{1}{3 b_{(4)}} \ln \left|u_{2} \mathcal{B}_{2}\right|^{2} \rightarrow x, \\
\varphi_{1}-\frac{1}{b_{(3)}} \ln \left|u_{2} \mathcal{B}_{2}\right|^{2} & \rightarrow \varphi_{1}, \quad \varphi_{2}+\frac{1}{b_{(3)}} \ln \left|u_{2} \mathcal{B}_{2}\right|^{2} \rightarrow \varphi_{2}, \\
\chi_{L}+\frac{1}{2} \sqrt{\frac{k}{6}} \ln \left(u_{2} \mathcal{B}_{2}\right) & \rightarrow \chi_{L}, \quad \chi_{R}+\frac{1}{2} \sqrt{\frac{k}{6}} \ln \left(\bar{u}_{2} \overline{\mathcal{B}}_{2}\right) \rightarrow \chi_{R} .
\end{aligned}
$$

The correlation function is now summarized as

$$
\left\langle\prod_{\nu=1}^{N} \Psi_{\nu}\left(z_{\nu}\right)\right\rangle=\left\langle\prod_{\nu=1}^{N} \mathcal{V}_{\nu}\left(z_{\nu}\right)\right\rangle
$$

where the action for the right hand side is

$$
\begin{aligned}
S= & \frac{1}{2 \pi} \int d^{2} w\left[\frac{3}{8} \partial x \bar{\partial} x+\frac{G_{i j}^{(3)}}{2} \partial x^{i} \bar{\partial} x^{j}-\frac{G_{i j}^{(3)}}{2} \partial \varphi^{i} \bar{\partial} \varphi^{j}-\partial \chi \bar{\partial} \chi\right] \\
& +\frac{1}{2 \pi} \int d^{2} w\left[\frac{1}{4} \sqrt{g} \mathcal{R}\left(Q_{x} x+\sum_{i=1}^{2} Q_{x, i} x^{i}-\sum_{i=1}^{2} Q_{\varphi, i} \varphi^{i}-Q_{\chi} \chi\right)+2 \pi \lambda \sum_{i=1}^{6} V_{i}\right] .
\end{aligned}
$$


The background charges are

$$
\begin{aligned}
& Q_{x}=\frac{3 b_{(4)}}{2}+\frac{3}{4 b_{(4)}}, \quad Q_{x, 1}=b_{(4)}+\frac{1}{b_{(4)}}, \quad Q_{x, 2}=b_{(4)}+\frac{1}{b_{(4)}}, \\
& Q_{\varphi, 1}=b_{(3)}, \quad Q_{\varphi, 2}=b_{(3)}+\frac{2}{b_{(3)}}, \quad Q_{\chi}=\sqrt{\frac{k}{6}}
\end{aligned}
$$

and the interaction terms are

$$
\begin{aligned}
& V_{1}=e^{-\frac{3}{4} x / b_{(4)}+\varphi^{1} / b_{(3)}+\sqrt{\frac{k}{6}}\left(\chi_{L}+\chi_{R}\right)}, \\
& V_{2}=e^{-\left(\frac{1}{4} x+\frac{1}{3} x_{1}+\frac{2}{3} x_{2}\right) / b_{(4)}+\varphi^{2} / b_{(3)}-\sqrt{\frac{k}{6}}\left(\chi_{L}+\chi_{R}\right)}, \\
& V_{3}=V_{0}^{\prime}=e^{\frac{3}{4} x / b_{(4)}+\varphi^{1} / b_{(3)}-\varphi^{2} / b_{(3)}-\sqrt{\frac{k}{6}}\left(\chi_{L}+\chi_{R}\right)}, \\
& V_{4}=e^{x_{1} / b_{(4)}} \\
& V_{5}=e^{x_{2} / b_{(4)}} \\
& V_{6}=e^{\left(\frac{1}{4} x-\frac{2}{3} x_{1}-\frac{1}{3} x_{2}\right) / b_{(4)}-\varphi^{1} / b_{(3)}+\varphi^{2} / b_{(3)}+\sqrt{\frac{k}{6}}\left(\chi_{L}+\chi_{R}\right) .}
\end{aligned}
$$

Here we have put $V_{0}^{\prime}$ back to the action. For $V_{4}, V_{5}$, we have used the self-duality of $\mathfrak{s l}(3)$ Toda field theory. The vertex operators are now of the form

$$
\begin{aligned}
\mathcal{V}_{\nu}\left(z_{\nu}\right)= & e^{\left(b_{(4)} j_{1}^{\nu}+\frac{3}{4 b_{(4)}}\right) x+\left(b_{(4)}\left(j_{2}^{\nu}-\frac{2}{3} j_{1}^{\nu}\right)+\frac{1}{b_{(4)}}\right) x_{1}+\left(b_{(4)}\left(j_{3}^{\nu}-\frac{1}{3} j_{1}^{\nu}\right)+\frac{1}{b_{(4)}}\right) x_{2}} \\
& \times e^{b_{(3)}\left(\left(l_{1}^{\nu}-\frac{2}{3 b_{(3)}^{2}}\right) \varphi_{1}+\left(l_{2}^{\nu}-\frac{4}{3 b_{(3)}^{2}}\right) \varphi_{2}\right)+2 \sqrt{\frac{6}{k}}\left(\left(m^{\nu}-\frac{k}{12}\right) \chi_{L}+\left(\bar{m}^{\nu}-\frac{k}{12}\right) \chi_{R}\right)} .
\end{aligned}
$$

In order to make the interaction terms to be of the form (2.17), we rotate fields and perform reflections as in the $N=2$ case. We first move from $\left(x, x_{1}, x_{2}\right)$ to $\left(\phi_{1}, \phi_{2}, \phi_{3}\right)$ by (5.31) and then perform the rotations of fields as

$$
\begin{gathered}
(k-4) \phi_{1}+(k-4) \phi_{2}+(k-3) \phi_{3}-\frac{1}{b_{(3)} b_{(4)}}\left(\varphi_{1}+\varphi_{2}\right) \rightarrow \phi_{1}, \\
\left(2-\frac{k}{2}\right) \phi_{1}+(k-3) \phi_{2}+\left(\frac{k}{2}-2\right) \phi_{3}+\frac{2}{3 b_{(3)} b_{(4)}}\left(\varphi_{1}-\varphi_{2}\right)-\sqrt{\frac{2 k}{3}} \frac{1}{b_{(4)}} \chi \rightarrow \phi_{2}, \\
\left(\frac{3 k}{2}-5\right) \phi_{1}+\left(\frac{k}{2}-2\right) \phi_{3}-\frac{1}{3 b_{(3)} b_{(4)}}\left(5 \varphi_{1}+\varphi_{2}\right)+\sqrt{\frac{2 k}{3}} \frac{1}{b_{(4)}} \chi \rightarrow \phi_{3}, \\
\frac{1}{b_{(3)} b_{(4)}}\left(\frac{1}{2} \phi_{1}+2 \phi_{2}+\frac{2}{3} \phi_{3}\right)+\left(1-\frac{k}{3}\right) \varphi_{1}+\left(6-\frac{5 k}{3}\right) \varphi_{2}-\sqrt{\frac{2 k}{3}} \frac{1}{b_{(3)}} \chi \rightarrow \varphi_{1}, \\
\frac{1}{2 b_{(3)} b_{(4)}}\left(3 \phi_{1}+\phi_{3}\right)+\left(6-\frac{5 k}{3}\right) \varphi_{1}+\left(1-\frac{k}{3}\right) \varphi_{2}+\sqrt{\frac{2 k}{3}} \frac{1}{b_{(3)}} \chi \rightarrow \varphi_{2}, \\
-\sqrt{\frac{k}{6}} \frac{1}{4 b_{(4)}}\left(\phi_{1}-2 \phi_{2}-\phi_{3}\right)+\sqrt{\frac{k}{6} \frac{1}{3 b_{(3)}}\left(\varphi_{1}-\varphi_{2}\right)+\left(1-\frac{k}{6}\right) \chi \rightarrow \chi .}
\end{gathered}
$$


The action is now

$$
\begin{aligned}
S= & \frac{1}{2 \pi} \int d^{2} w\left[\frac{G_{i j}^{(4)}}{2} \partial \phi^{i} \bar{\partial} \phi^{j}-\frac{G_{i j}^{(3)}}{2} \partial \varphi^{i} \bar{\partial} \varphi^{j}-\partial \chi \bar{\partial} \chi\right] \\
& +\frac{1}{2 \pi} \int d^{2} w\left[\frac{1}{4} \sqrt{g} \mathcal{R}\left(\sum_{i=1}^{3} b_{(4)} \phi^{i}-\sum_{i=1}^{2} b_{(3)} \varphi^{i}\right)+2 \pi \lambda \sum_{i=1}^{6} \tilde{V}_{i}\right]
\end{aligned}
$$

with interaction terms

$$
\begin{aligned}
& \tilde{V}_{1}=V_{1}=e^{\phi^{1} / b_{(4)}-\varphi^{1} / b_{(3)}+\sqrt{\frac{k}{6}}\left(\chi_{L}+\chi_{R}\right)}, \\
& \tilde{V}_{2}=e^{\left(\phi^{1}-\phi^{2}\right) / b_{(4)}+\varphi^{1} / b_{(3)}-\sqrt{\frac{k}{6}}\left(\chi_{L}+\chi_{R}\right)}, \\
& \tilde{V}_{3}=e^{\left(\phi^{2}-\phi^{1}\right) / b_{(4)}+\left(\varphi^{1}-\varphi^{2}\right) / b_{(3)}+\sqrt{\frac{k}{6}}\left(\chi_{L}+\chi_{R}\right)}, \\
& \tilde{V}_{4}=V_{6}=e^{\left(\phi^{2}-\phi^{3}\right) / b_{(4)}+\left(\varphi^{2}-\varphi^{1}\right) / b_{(3)}-\sqrt{\frac{k}{6}}\left(\chi_{L}+\chi_{R}\right)}, \\
& \tilde{V}_{5}=e^{\left(\phi^{3}-\phi^{2}\right) / b_{(4)}+\varphi^{2} / b_{(3)}+\sqrt{\frac{k}{6}}\left(\chi_{L}+\chi_{R}\right)}, \\
& \tilde{V}_{6}=V_{2}=e^{\phi^{3} / b_{(4)}-\varphi^{2} / b_{(3)}-\sqrt{\frac{k}{6}}\left(\chi_{L}+\chi_{R}\right)} .
\end{aligned}
$$

Here $\tilde{V}_{2}, \tilde{V}_{3}, \tilde{V}_{5}$ are obtained from $V_{5}, V_{4}, V_{3}$, respectively, by performing certain reflection relations as before. This form of interaction terms is the same as that in (2.17) as desired. Similarly, the vertex operators can be put in a nice form as

$$
\mathcal{V}_{\nu}\left(z_{\nu}\right)=e^{b_{(4)}\left(j_{3}^{\nu} \phi_{1}+j_{2}^{\nu} \phi_{2}+j_{1}^{\nu} \phi_{3}\right)+b_{(3)}\left(l_{2}^{\nu} \varphi_{1}+l_{1}^{\nu} \varphi_{2}\right)+2 \sqrt{\frac{6}{k}}\left(m^{\nu} \chi_{L}+\bar{m}^{\nu} \chi_{R}\right)}
$$

by applying a sequence of reflection relations.

\section{Conclusion and discussions}

In this paper, we generalized the FZZ-duality by replacing (1.1) with a higher rank coset (1.3). A proof of the original FZZ-duality was given by applying the reduction method of [27]. For the higher rank generalizations, we made use of an extended version of the reduction method recently developed in [33]. We first find out an free field realization of the coset algebra by following the analysis of [39, 40]. We then applied the reduction method to the coset model for $N=2,3$ and derived the higher rank FZZ-duality between the coset model and the theory with an $\mathfrak{s l}(N+1 \mid N)$ structure. For the $N=3$ case, we adopted a technique of [33] by exchanging the free field realizations of BP-algebra. During the process, we applied the self-duality of Toda field theory, which was the key point to derive strong/weak dualities.

The theory dual to the coset model (1.3) with generic $N$ should be given by

$$
\begin{aligned}
S= & \frac{1}{2 \pi} \int d^{2} w\left[\frac{G_{i j}^{(N+1)}}{2} \partial \phi^{i} \bar{\partial} \phi^{j}-\frac{G_{i j}^{(N)}}{2} \partial \varphi^{i} \bar{\partial} \varphi^{j}-\partial \chi \bar{\partial} \chi\right] \\
& +\frac{1}{2 \pi} \int d^{2} w\left[\frac{1}{4} \sqrt{g} \mathcal{R}\left(\sum_{j=1}^{N} b_{(N+1)} \phi^{j}-\sum_{j=1}^{N-1} b_{(N)} \varphi^{j}\right)+2 \pi \lambda \sum_{l=1}^{2 N} V_{l}\right]
\end{aligned}
$$


where the interaction terms are as in (2.17). We can see that the theory reduces to the one obtained in this paper for $N=2,3$ (and also the sine-Liouville theory for $N=1$ if we use $\left.\tilde{X}=\tilde{X}_{L}-\tilde{X}_{R}=-i\left(\chi_{L}+\chi_{R}\right)\right)$. It is an important open problem to give a proof of the higher rank FZZ-dualities. We expect that a generalized version of the technique utilized for $N=3$ is necessary in order to achieve this.

We would like to consider the following open problems and future directions. Firstly, we have not discussed the spectra of coset theories and, for instance, we have not specified the regions for the parameters appearing in (3.14). The information was not used for the comparison of correlation functions. However, the problem of spectra itself is quite important, and in particular, we would like to know whether unitary theories can be constructed from the coset theories or not. Furthermore, our construction of coset theories may not be well formulated as discussed in footnotes 5 and 6 . We would like to derive our expressions of coset theories as gauge fixed forms of gauged WZNW models. In [26], the correspondence among correlation functions was derived even including all pre-factors. It might be useful to give a derivation of duality with keeping all pre-factors in the current case as well. A non-trivial point may arise from the map between the free field realizations of BP-algebra. We would like to examine the theory with BP-algebra symmetry furthermore in order to understand the map more closely.

In appendix $\mathrm{A}$, we discuss an $\mathcal{N}=2$ supersymmetric version of our duality. We would like to extend the current analysis to the supersymmetric case as mentioned in the introduction. The duality might be derived by following the analysis of [51], where the $\mathcal{N}=2$ supersymmetric version of the original FZZ-duality was proven as a mirror symmetry. This version of duality was utilized to examine singular Calabi-Yau geometry [52, 53], and its higher rank generalizations may be useful for similar purposes. In this paper, we have examined correlation functions only on a Riemann sphere. On the other hand, the FZZ-duality was extended for higher genus Riemann surfaces in [26]. Similar extensions would be straightforward even for higher rank cases. Moreover, the FZZ-duality was generalized for the worldsheet of disk topology in [54]. However, it seems to be difficult to extend the analysis for higher rank cases, since we do not know much about boundary Toda-like field theories.

Our results can be viewed as a conformal field theoretic version of special cases of duality of $Y_{N_{1}, N_{2}, N_{3}}$-algebras where the first two labels are interchanged. These algebras however enjoy a triality and it is of course desirable to get a conformal field theoretic derivation of the complete triality at least in certain low rank cases. We aim to address this issue in future work.

An important and quite complicated open question are dualities involving the small and large $\mathcal{N}=4$ superconformal algebras. Note that the small and large $\mathcal{N}=4$ superconformal algebras coincide with certain cosets involving $\mathfrak{d}(2,1 ; \alpha)$ (and $\mathfrak{p s l}(2 \mid 2)$ ) at level one [55] and the question is if one can derive $\mathcal{N}=4$ superconformal field theories from WZNW theories of $\mathfrak{d}(2,1 ; \alpha)$ (and $\mathfrak{p s l}(2 \mid 2))$ at level one. This is relevant for the AdS/CFT correspondence which is a an interesting example of strong/weak duality, namely string theory on strongly curved AdS space corresponds to weakly coupled CFT. Recently, it was argued that "tensionless" superstrings on $\mathrm{AdS}_{3} \times \mathrm{S}^{3} \times \mathrm{T}^{4}$ with one unit of NSNS-flux is dual to the undeformed symmetric orbifold of $\mathrm{T}^{4}$, see, e.g., [56-58]. The match of correla- 
tion functions was confirmed recently in [59-62], and, in particular, the reduction method of $[26,27$ from $\mathfrak{s l}(2)$ WZNW model to Liouville field theory was utilized in [61]. Some modifications of the higher rank generalizations developed in [33] and this paper might be useful for investigating the AdS/CFT correspondence involving higher dimensional AdS strings. Higher spin gravity may be able to describe (a part of) tensionless strings. The analysis in this paper would be useful to study CFT dual to higher spin gravity as mentioned in the introduction. Making use of higher spin holography with $\mathcal{N}=4$ supersymmetry [63, 64] or $\mathcal{N}=3$ supersymmetry $[8,65,66]$, we would like to examine possible relations between superstrings and higher spin gravity.

\section{Acknowledgments}

We are grateful to N. Genra for useful discussions. The work of TC is supported by NSERC Grant Number RES0048511. The work of YH is supported by JSPS KAKENHI Grant Number 16H02182 and 19H01896.

\section{A Supersymmetric dualities}

In this appendix, we explain an $\mathcal{N}=2$ supersymmetric version of our higher rank dualities. We consider the Kazama-Suzuki model $[67,68]$ of the form

$$
\frac{\mathfrak{s l}(N+1)_{k} \times \mathfrak{s o}(2 N)_{1}}{\mathfrak{s l}(N)_{k-1} \times \mathfrak{u}(1)}
$$

which can be regarded as the $\mathcal{N}=2$ supersymmetric extension of the coset model (1.3). The symmetry of the coset model is believed to be the $\mathcal{N}=2 \mathrm{~W}_{N+1}$-algebra $[22,23]$. From this, we can expect that the coset model (A.1) is dual to the $\mathfrak{s l}(N+1 \mid N)$ Toda field theory with the same symmetry algebra. In the following, we provide screening charges for the $\mathcal{N}=2 \mathrm{~W}_{N+1}$-algebra, which correspond to interaction terms of the Toda field theory.

We can choose a purely odd simple root system for $\mathfrak{s l}(N+1 \mid N)$ Lie superalgebra. We may introduce two orthogonal bases $\epsilon_{j}(j=1,2, \ldots, N+1)$ and $\delta_{j}(j=1,2, \ldots, N)$ satisfying

$$
\epsilon_{i} \cdot \epsilon_{j}=\delta_{i, j}, \quad \delta_{i} \cdot \delta_{j}=-\delta_{i, j} .
$$

The odd simple roots are expressed as

$$
\alpha_{2 j-1}=\epsilon_{j}-\delta_{j}, \quad \alpha_{2 j}=\delta_{j}-\epsilon_{j+1}
$$

with $j=1,2, \ldots, N$.

We introduce free bosons $x_{a}$ and free fermions $\psi_{a}$ satisfying

$$
x_{a}(z) x_{b}(0) \sim-\delta_{a, b} \ln z, \quad \psi_{a}(z) \psi_{b}(0) \sim \frac{\delta_{a, b}}{z} .
$$

There are background charges for the bosonic fields such that screening charges are of dimension one. We prepare $x_{a}, \psi_{a}$ with $a, b=1,2, \ldots, 2 N+1$ but a pair of certain combination decouples at the end. Screening operators are now expressed as [22, 23]

$$
V_{l}=\alpha_{l} \cdot \psi e^{\alpha_{l} \cdot x / b_{(N+1)}}
$$

with $l=1,2, \ldots, 2 N$ and $b_{(N+1)}$ given in (2.18) with $a=N+1$. 
We may redefine the bosonic fields by

$$
\begin{aligned}
\phi_{i} & =\left(\epsilon_{i}-\epsilon_{i+1}\right) \cdot x & (i=1,2, \ldots, N), \\
\varphi_{i} & =\left(\delta_{i}-\delta_{i+1}\right) \cdot x & (i=1,2, \ldots, N-1), \\
\chi & =\frac{1}{N+1} \sum_{i=1}^{N+1} \epsilon_{i} \cdot x-\frac{1}{N} \sum_{i=1}^{N} \delta_{i} \cdot x . &
\end{aligned}
$$

We can check that they are orthogonal to the decoupled mode, $\sum_{i=1}^{N+1} \epsilon_{i} \cdot x-\sum_{i=1}^{N} \delta_{i} \cdot x$. The OPEs among these fields are given by (2.16). The screening operators are now expressed as

$$
\begin{aligned}
V_{1} & =\alpha_{1} \cdot \psi e^{\left(\phi^{1}-\varphi^{1}+\chi\right) / b_{(N+1)}}, \\
V_{2} & =\alpha_{2} \cdot \psi e^{\left(\phi^{1}-\phi^{2}+\varphi^{1}-\chi\right) / b_{(N+1)}}, \\
V_{3} & =\alpha_{3} \cdot \psi e^{\left(\phi^{2}-\phi^{1}+\varphi^{1}-\varphi^{2}+\chi\right) / b_{(N+1)}}, \\
V_{4} & =\alpha_{4} \cdot \psi e^{\left(\phi^{2}-\phi^{3}+\varphi^{2}-\varphi^{1}-\chi\right) / b_{(N+1)}}, \\
\vdots & \\
V_{2 N-1}= & \alpha_{2 N-1} \cdot \psi e^{\left(\phi^{N}-\phi^{N-1}+\varphi^{N-1}+\chi\right) / b_{(N+1)}}, \\
V_{2 N}= & \alpha_{2 N} \cdot \psi e^{\left(\phi^{N}-\varphi^{N-1}-\chi\right) / b_{(N+1)}} .
\end{aligned}
$$

\section{B Free field realizations of BP-algebra}

In this appendix, we summarize the results in section 2 of [33] on the BP-algebra and its free field realizations. The BP-algebra is generated by a spin-one current $H(z)$, two spin$3 / 2$ bosonic currents $G^{ \pm}(z)$, and a spin-two current $T(z)$, which is the energy momentum tensor. The non-trivial OPEs among them are [36]

$$
\begin{array}{rlrl}
T(z) T(0) & \sim \frac{\frac{1}{2} c}{z^{4}}+\frac{2 T(0)}{z^{2}}+\frac{\partial T(0)}{z}, & \\
T(z) G^{ \pm}(0) & \sim \frac{\frac{3}{2} G^{ \pm}(0)}{z^{2}}+\frac{\partial G^{ \pm}(0)}{z}, & T(z) H(0) \sim \frac{H(0)}{z^{2}}+\frac{\partial H(0)}{z}, \\
H(z) H(0) & \sim-\frac{\frac{1}{3}(2 k-3)}{z^{2}}, & H(z) G^{ \pm}(0) \sim \pm \frac{G^{ \pm}(0)}{z}, \quad(\mathrm{~B} .1) \\
G^{+}(z) G^{-}(0) & \sim \frac{(k-1)(2 k-3)}{z^{3}}-\frac{3(k-1) H(0)}{z^{2}} & \\
& +\frac{3 H H(0)+(k-3) T(0)-\frac{3}{2}(k-1) \partial H(0)}{z} .
\end{array}
$$

The central charge is

$$
c=6(k-3)+25+\frac{24}{k-3} .
$$

In order to realize the algebra in terms of free fields, we prepare two free bosons $x_{j}$ $(j=1,2)$ and a ghost system $(\gamma, \beta)$ satisfying

$$
x_{i}(z) x_{j}(0) \sim-G_{i j}^{(3)} \ln z, \quad \gamma(z) \beta(0) \sim \frac{1}{z} .
$$


There are two types of screening charges for the same BP-algebra. A type of screening charges are given by [36]

$$
Q_{1}=\oint d w e^{b_{(3)} x_{1}} \beta, \quad Q_{2}=\oint d w e^{b_{(3)} x_{2}} \gamma .
$$

The generators should commute with these screening charges, and in particular, the spinone current is

$$
H=\frac{1}{b_{(3)}}\left(\partial x^{2}-\partial x^{1}\right)-\gamma \beta .
$$

The index of $x_{j}$ is raised by $G^{(3) i j}$, where the expression of $G^{(3) i j}$ can be found in (3.3). Another type of screening charges are [33, 37, 38]

$$
Q_{1}=\oint d w e^{b_{(3)} x_{1}} \beta, \quad Q_{2}=\oint d w e^{b_{(3)} x_{2}} .
$$

In this case, the spin-one current is obtained as

$$
H=\frac{1}{b_{(3)}} \partial x^{1}+\gamma \beta \text {. }
$$

In the main context, the energy momentum tensor is given by the twisted one

$$
T_{t}(z)=T(z)+\frac{1}{2} \partial H(z) .
$$

With respect to $T_{t}(z)$, the conformal dimensions of $G^{+}(z)$ and $G^{-}(z)$ become one and two, respectively. In particular, the generators have integer modded expansions as

$$
\begin{aligned}
T_{t}(z) & =\sum_{n \in \mathbb{Z}} \frac{L_{n}}{z^{n+2}}, \quad H(z)=\sum_{n \in \mathbb{Z}} \frac{H_{n}}{z^{n+1}}, \\
G^{+}(z) & =\sum_{n \in \mathbb{Z}} \frac{G_{n}^{+}}{z^{n+1}}, \quad G^{-}(z)=\sum_{n \in \mathbb{Z}} \frac{G_{n}^{-}}{z^{n+2}} .
\end{aligned}
$$

Primary states with respect to the algebra are defined such as to satisfy the conditions

$$
L_{n}\left|c_{2}, c_{3}, m\right\rangle=G_{n}^{ \pm}\left|c_{2}, c_{3}, m\right\rangle=H_{n}\left|c_{2}, c_{3}, m\right\rangle=0
$$

for $n=1,2,3, \ldots$. The representation of the primary state is labeled by the eigenvalues of the second-order and third-order Casimir operators denoted as $c_{2}, c_{3}$. Here the second-order Casimir operator is given by $L_{0}$ and the expression of the third-order Casimir operator can be found in [33]. The other parameter $m$ is defined by

$$
H_{0}\left|c_{2}, c_{3}, m\right\rangle=m\left|c_{2}, c_{3}, m\right\rangle
$$

and in particular

$$
G_{0}^{ \pm}\left|c_{2}, c_{3}, m\right\rangle \propto\left|c_{2}, c_{3}, m \pm 1\right\rangle .
$$

In terms of free fields, the primary states may be expressed by vertex operators as

$$
\left|c_{2}, c_{3}, m\right\rangle \propto \lim _{z \rightarrow 0} V_{j_{1}, j_{2}, \alpha}(z)|0\rangle, \quad V_{j_{1}, j_{2}, \alpha}(z)=\gamma^{\alpha} e^{b_{(3)}\left(j_{1} \phi_{1}+j_{2} \phi_{2}\right)} .
$$


The parameters $c_{2}, c_{3}$ are independent of both of $\alpha$ and the choice of free field realizations, see [33] for explicit expressions. The other parameter $m$ depends on both of $\alpha$ and the choice of free field realizations. For the free field realization with (B.4), the eigenvalue of $H$ is given by

$$
m=-j_{2}+j_{1}+\alpha .
$$

For the other realization with (B.6), the eigenvalue of $H$ is

$$
m=-j_{1}-\alpha .
$$

These results imply that we should replace $\alpha \leftrightarrow-2 j_{1}+j_{2}-\alpha$ when we exchange the free field realizations.

Open Access. This article is distributed under the terms of the Creative Commons Attribution License (CC-BY 4.0), which permits any use, distribution and reproduction in any medium, provided the original author(s) and source are credited.

\section{References}

[1] V. Fateev, A. Zamolodchikov and A. Zamolodchikov, unpublished.

[2] E. Witten, On string theory and black holes, Phys. Rev. D 44 (1991) 314 [INSPIRE].

[3] V. Kazakov, I.K. Kostov and D. Kutasov, A matrix model for the two-dimensional black hole, Nucl. Phys. B 622 (2002) 141 [hep-th/0101011] [InSPIRE].

[4] T. Creutzig and A.R. Linshaw, Trialities of $\mathcal{W}$-algebras, arXiv:2005.10234 [INSPIRE].

[5] L.F. Alday, D. Gaiotto and Y. Tachikawa, Liouville correlation functions from four-dimensional gauge theories, Lett. Math. Phys. 91 (2010) 167 [arXiv:0906.3219] [INSPIRE].

[6] N. Wyllard, $A_{N-1}$ conformal Toda field theory correlation functions from conformal $N=2$ $\mathrm{SU}(N)$ quiver gauge theories, JHEP 11 (2009) 002 [arXiv:0907.2189] [INSPIRE].

[7] M.R. Gaberdiel and R. Gopakumar, An AdS $S_{3}$ dual for minimal model CFTs, Phys. Rev. D 83 (2011) 066007 [arXiv: 1011.2986] [INSPIRE].

[8] T. Creutzig, Y. Hikida and P.B. Ronne, Higher spin AdS $S_{3}$ supergravity and its dual CFT, JHEP 02 (2012) 109 [arXiv:1111.2139] [InSPIRE].

[9] D. Gaiotto and M. Rapčák, Vertex algebras at the corner, JHEP 01 (2019) 160 [arXiv: 1703.00982] [INSPIRE].

[10] V.G. Kac and M. Wakimoto, Quantum reduction and representation theory of superconformal algebras, math-ph/0304011 [INSPIRE].

[11] B. Feigin and E. Frenkel, Duality in W-algebras, Int. Math. Res. Not. 1991 (1991) 75.

[12] T. Arakawa, T. Creutzig and A.R. Linshaw, $W$-algebras as coset vertex algebras, Invent. Math. 218 (2019) 145 [arXiv: 1801.03822] [INSPIRE].

[13] B.L. Feigin and A.M. Semikhatov, $\mathcal{W}_{n}^{(2)}$ algebras, Nucl. Phys. B 698 (2004) 409 [math.QA/0401164] [INSPIRE].

[14] T. Creutzig, N. Genra and S. Nakatsuka, Duality of subregular $W$-algebras and principal $W$-superalgebras, arXiv:2005.10713 [INSPIRE]. 
[15] T. Creutzig and D. Gaiotto, Vertex algebras for S-duality, Commun. Math. Phys. 379 (2020) 785 [arXiv: 1708.00875] [INSPIRE].

[16] E. Frenkel and D. Gaiotto, Quantum Langlands dualities of boundary conditions, D-modules, and conformal blocks, Commun. Num. Theor. Phys. 14 (2020) 199 [arXiv:1805.00203] [INSPIRE].

[17] T. Creutzig, D. Gaiotto and A.R. Linshaw, S-duality for the large $N=4$ superconformal algebra, Commun. Math. Phys. 374 (2020) 1787 [arXiv: 1804.09821] [INSPIRE].

[18] M. Bershtein, B. Feigin and G. Merzon, Plane partitions with a "pit": generating functions and representation theory, Selecta Math. 24 (2018) 21.

[19] A. Litvinov and L. Spodyneiko, On W-algebras commuting with a set of screenings, JHEP 11 (2016) 138 [arXiv:1609.06271] [INSPIRE].

[20] T. Procházka and M. Rapčák, $\mathcal{W}$-algebra modules, free fields, and Gukov-Witten defects, JHEP 05 (2019) 159 [arXiv: 1808.08837] [INSPIRE].

[21] M. Rapčák, Y. Soibelman, Y. Yang and G. Zhao, Cohomological Hall algebras, vertex algebras and instantons, Commun. Math. Phys. 376 (2019) 1803 [arXiv:1810.10402] [INSPIRE].

[22] K. Ito, Quantum Hamiltonian reduction and $N=2$ coset models, Phys. Lett. B 259 (1991) 73 [INSPIRE].

[23] K. Ito, $N=2$ superconformal $C P_{n}$ model, Nucl. Phys. B 370 (1992) 123 [INSPIRE].

[24] N. Genra and A.R. Linshaw, Ito's conjecture and the coset construction for $\mathcal{W}^{k}(\mathfrak{s l}(3 \mid 2))$, arXiv:1901.02397 [INSPIRE].

[25] T. Creutzig and A.R. Linshaw, Cosets of affine vertex algebras inside larger structures, J. Algebra 517 (2019) 396 [arXiv:1407.8512] [INSPIRE].

[26] Y. Hikida and V. Schomerus, The FZZ-duality conjecture: a proof, JHEP 03 (2009) 095 [arXiv:0805.3931] [INSPIRE].

[27] Y. Hikida and V. Schomerus, $H_{3}^{+}$WZNW model from Liouville field theory, JHEP 10 (2007) 064 [arXiv: 0706.1030] [INSPIRE].

[28] S. Ribault and J. Teschner, $H_{3}^{+}-W Z N W$ correlators from Liouville theory, JHEP 06 (2005) 014 [hep-th/0502048] [INSPIRE].

[29] S. Ribault, Knizhnik-Zamolodchikov equations and spectral flow in $A_{d S_{3}}$ string theory, JHEP 09 (2005) 045 [hep-th/0507114] [INSPIRE].

[30] Y. Hikida and V. Schomerus, Structure constants of the OSP(1|2) WZNW model, JHEP 12 (2007) 100 [arXiv:0711.0338] [INSPIRE].

[31] T. Creutzig, Y. Hikida and P.B. Ronne, Supergroup-extended super Liouville correspondence, JHEP 06 (2011) 063 [arXiv:1103.5753] [INSPIRE].

[32] T. Creutzig, Y. Hikida and P.B. Rønne, Correspondences between WZNW models and CFTs with $W$-algebra symmetry, JHEP 02 (2016) 048 [arXiv: 1509.07516] [INSPIRE].

[33] T. Creutzig, N. Genra, Y. Hikida and T. Liu, Correspondences among CFTs with different $W$-algebra symmetry, Nucl. Phys. B 957 (2020) 115104 [arXiv: 2002.12587] [INSPIRE].

[34] S. Ribault, On sl $l_{3}$ Knizhnik-Zamolodchikov equations and $W_{3}$ null-vector equations, JHEP 10 (2009) 002 [arXiv:0811.4587] [INSPIRE]. 
[35] A.M. Polyakov, Gauge transformations and diffeomorphisms, Int. J. Mod. Phys. A 5 (1990) 833 [INSPIRE].

[36] M. Bershadsky, Conformal field theories via Hamiltonian reduction, Commun. Math. Phys. 139 (1991) 71 [INSPIRE].

[37] N. Genra, Screening operators for $W$-algebras, arXiv:1606.00966 [INSPIRE].

[38] N. Genra, Screening operators and parabolic inductions for affine $\mathcal{W}$-algebras, arXiv: 1806.04417.

[39] A. Gerasimov, A. Marshakov and A. Morozov, Free field representation of parafermions and related coset models, Nucl. Phys. B 328 (1989) 664 [InSPIRE].

[40] M. Kuwahara, N. Ohta and H. Suzuki, Conformal field theories realized by free fields, Nucl. Phys. B 340 (1990) 448 [INSPIRE].

[41] A.R. Linshaw, Universal two-parameter $\mathcal{W}_{\infty}$-algebra and vertex algebras of type $\mathcal{W}(2,3, \ldots, N)$, arXiv: 1710.02275 [INSPIRE].

[42] M.R. Gaberdiel and R. Gopakumar, Triality in minimal model holography, JHEP 07 (2012) 127 [arXiv: 1205.2472] [INSPIRE].

[43] T. Procházka, Exploring $\mathcal{W}_{\infty}$ in the quadratic basis, JHEP 09 (2015) 116 [arXiv: 1411.7697] [INSPIRE].

[44] T. Procházka and M. Rapčák, Webs of W-algebras, JHEP 11 (2018) 109 [arXiv: 1711.06888] [INSPIRE].

[45] T. Procházka, $\mathcal{W}$-symmetry, topological vertex and affine Yangian, JHEP 10 (2016) 077 [arXiv: 1512.07178] [INSPIRE].

[46] D. Kutasov and N. Seiberg, More comments on string theory on AdS $S_{3}$, JHEP 04 (1999) 008 [hep-th/9903219] [INSPIRE].

[47] P. Di Francesco, P. Mathieu and D. Senechal, Conformal field theory, Springer-Verlag, New York, NY, U.S.A. (1997) [INSPIRE].

[48] P. Goddard, A. Kent and D.I. Olive, Unitary representations of the Virasoro and super-Virasoro algebras, Commun. Math. Phys. 103 (1986) 105 [INSPIRE].

[49] J.M. Maldacena and H. Ooguri, Strings in $A d S_{3}$ and the $\mathrm{SL}(2, R)$ WZW model. Part 3. Correlation functions, Phys. Rev. D 65 (2002) 106006 [hep-th/0111180] [INSPIRE].

[50] R. Argurio, A. Giveon and A. Shomer, Superstrings on $A d S_{3}$ and symmetric products, JHEP 12 (2000) 003 [hep-th/0009242] [INSPIRE].

[51] K. Hori and A. Kapustin, Duality of the fermionic $2 D$ black hole and $N=2$ Liouville theory as mirror symmetry, JHEP 08 (2001) 045 [hep-th/0104202] [INSPIRE].

[52] H. Ooguri and C. Vafa, Two-dimensional black hole and singularities of CY manifolds, Nucl. Phys. B 463 (1996) 55 [hep-th/9511164] [INSPIRE].

[53] A. Giveon, D. Kutasov and O. Pelc, Holography for noncritical superstrings, JHEP 10 (1999) 035 [hep-th/9907178] [INSPIRE].

[54] T. Creutzig, Y. Hikida and P.B. Ronne, The FZZ duality with boundary, JHEP 09 (2011) 004 [arXiv: 1012.4731] [INSPIRE].

[55] T. Creutzig, B. Feigin and A.R. Linshaw, $N=4$ superconformal algebras and diagonal cosets, Int. Math. Res. Not. (2020) rnaa078 [arXiv:1910.01228] [INSPIRE]. 
[56] M.R. Gaberdiel and R. Gopakumar, Tensionless string spectra on $A d S_{3}$, JHEP 05 (2018) 085 [arXiv: 1803. 04423] [INSPIRE].

[57] L. Eberhardt, M.R. Gaberdiel and R. Gopakumar, The worldsheet dual of the symmetric product CFT, JHEP 04 (2019) 103 [arXiv: 1812.01007] [INSPIRE].

[58] G. Giribet, C. Hull, M. Kleban, M. Porrati and E. Rabinovici, Superstrings on $A d S_{3}$ at $k=1$, JHEP 08 (2018) 204 [arXiv: 1803. 04420] [INSPIRE].

[59] L. Eberhardt, M.R. Gaberdiel and R. Gopakumar, Deriving the AdS $S_{3} / C F T_{2}$ correspondence, JHEP 02 (2020) 136 [arXiv: 1911.00378] [INSPIRE].

[60] L. Eberhardt, $A d S_{3} / C F T_{2}$ at higher genus, JHEP 05 (2020) 150 [arXiv:2002.11729] [INSPIRE].

[61] Y. Hikida and T. Liu, Correlation functions of symmetric orbifold from $A d S_{3}$ string theory, JHEP 09 (2020) 157 [arXiv:2005.12511] [INSPIRE].

[62] A. Dei, M.R. Gaberdiel, R. Gopakumar and B. Knighton, Free field world-sheet correlators for $A d S_{3}$, arXiv: 2009.11306 [INSPIRE].

[63] M.R. Gaberdiel and R. Gopakumar, Large $N=4$ holography, JHEP 09 (2013) 036 [arXiv: 1305.4181] [INSPIRE].

[64] M.R. Gaberdiel and R. Gopakumar, Higher spins \& strings, JHEP 11 (2014) 044 [arXiv: 1406.6103] [INSPIRE].

[65] T. Creutzig, Y. Hikida and P.B. Ronne, Extended higher spin holography and Grassmannian models, JHEP 11 (2013) 038 [arXiv:1306.0466] [INSPIRE].

[66] T. Creutzig, Y. Hikida and P.B. Ronne, Higher spin AdS $S_{3}$ holography with extended supersymmetry, JHEP 10 (2014) 163 [arXiv:1406.1521] [INSPIRE].

[67] Y. Kazama and H. Suzuki, Characterization of $N=2$ superconformal models generated by coset space method, Phys. Lett. B 216 (1989) 112 [INSPIRE].

[68] Y. Kazama and H. Suzuki, New $N=2$ superconformal field theories and superstring compactification, Nucl. Phys. B 321 (1989) 232 [INSPIRE]. 